\title{
Acoustic Features of Infant-Directed Speech to Infants with Hearing Loss
}

\author{
Irena Lovcevic $^{1, a), ~ b}$, Marina Kalashnikova ${ }^{2}$, and Denis Burnham ${ }^{1}$

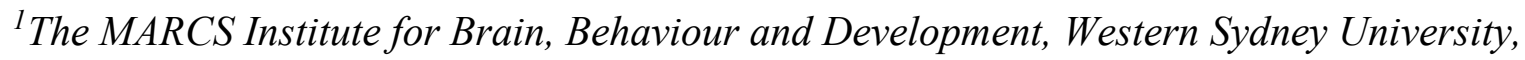 \\ Locked Bag 1797, Penrith NSW 2751, Australia \\ ${ }^{2}$ BCBL. Basque Center on Cognition, Brain and Language, Mikeletegi Pasealekua, 69,
} Donostia, Gipuzkoa 20009, Spain

a Electronic mail: irena.lovcevic@ircn.jp

${ }^{b}$ Current address: International Research Center for Neurointelligence (IRCN), The University of Tokyo, 7-3-1 Hongo Bunkyo-ku, Tokyo, 113-0033 Japan 


\begin{abstract}
This study investigated the effects of hearing loss and hearing experience on the acoustic features of infant-directed speech (IDS) to infants with hearing loss (HL) compared to controls with normal hearing $(\mathrm{NH})$ matched by either chronological or hearing age (Experiment 1) and across development in infants with HL as well as the relation between IDS features and infants' developing lexical abilities (Experiment 2). Both experiments included detailed acoustic analyses of mothers' productions of the three corner vowels $/ \mathrm{a}, \mathrm{i}, \mathrm{u} /$ and utterance-level pitch in IDS and in adult-directed speech (ADS). Experiment 1 demonstrated that IDS to infants with HL was acoustically more variable than IDS to hearing-age matched infants with NH. Experiment 2 yielded no changes in IDS features over development; however, the results did show a positive relationship between formant distances in mothers' speech and infants' concurrent receptive vocabulary size, as well as between vowel hyperarticulation and infants' expressive vocabulary. These findings suggest that despite their HL and thus diminished access to speech input, infants with HL are exposed to IDS with generally similar acoustic qualities as are infants with NH. However, some differences persist, indicating that infants with HL might receive less intelligible speech.
\end{abstract}




\section{INTRODUCTION}

Approximately two out of every 1000 infants worldwide are born with unilateral or bilateral hearing loss (HL) (van Wieringen, Boudewyns, Sangen, Wouters, \& Desloovere, 2019). Infants with congenital HL have limited access to auditory input both before and after birth (Moeller \& Tomblin, 2015), and the nature of their acoustic input is degraded since it is significantly different from the sound conducted through a normally functioning ear (Macherey \& Carlyon, 2014; Zeng, 2004; Zeng, Tang, \& Lu, 2014). However, after birth, this early deprivation can be mediated by Hearing Aids or Cochlear Implants, which may facilitate infants' early access to linguistic input, which plays a fundamental role in their early language development. However, there is no clear indication whether the quality (Lam \& Kitamura, 2010; Wieland, Burnham, Kondaurova, Bergeson, \& Dilley, 2015) and quantity (Vanormelingen, De Maeyer, \& Gillis, 2016) of the early linguistic input to infants with HL differs from their peers with NH. This study investigates this issue by focusing on the acoustic features of speech directed to infants with HL as a function of their age and hearing experience.

When addressing infants, adults produce a special speech register known as infant-directed speech (IDS) (Fernald \& Simon, 1984), which can be differentiated from adult-directed speech (ADS) by a number of linguistic, acoustic, and visual properties (Chong, Werker, Russell, \& Carroll, 2003; Cooper \& Aslin, 1990; Fernald \& Simon, 1984; Kalashnikova, Carignan, \& Burnham, 2017; Kuhl et al., 1997). Two acoustic properties of IDS, namely heightened pitch and acoustic exaggeration of vowels have been proposed to serve a role in facilitating infants' language acquisition. These two IDS properties will be the focus of this study. Exaggerated pitch in IDS refers to the increases in mean height and range of fundamental frequency (F0) in IDS compared to ADS (Fernald \& Simon, 1984; Fernald et al., 1989; Trainor, Austin, \& Desjardins, 2000). Vowel hyperarticulation refers to the greater vowel space area encompassed by the three corner vowels $(/ \mathrm{i}, \mathrm{u}, \mathrm{a} /)$ in IDS compared to ADS. It is indexed by plotting these three vowels in two-dimensional Formant 1 (F1) / Formant 2 (F2) space and 
calculating the area of the resulting triangle. Using this measure, studies have shown that the vowel triangle area for IDS is larger than for ADS (Burnham, Kitamura, \& Vollmer-Conna, 2002; Kalashnikova et al., 2017; Kuhl et al., 1997; Uther, Knoll, \& Burnham, 2007). It is noteworthy that these properties have been identified in IDS produced by English-speaking parents, and the extent to which they manifest in languages other than English continues to be debated. For instance, heightened pitch has been found in French, Italian, German (Fernald, 1989), Mandarin (Grieser \& Kuhl, 1988), Cantonese (Rattanasone, Burnham, \& Reilly, 2013), Thai (Kitamura, Thanavishuth, Burnham, \& Luksaneeyanawin, 2002), and Japanese (Fernald, 1989), but not in Quiche Mayan (Bernstein Ratner \& Pye, 1984), which may be due to cultural differences in the use of pitch in that society. Vowel hyperarticulation has been reported for IDS in Mandarin Chinese (Liu, Kuhl, \& Tsao, 2003; Tang, Xu Rattanasone, Yuen, \& Demuth, 2017) and Japanese (Miyazawa, Shinya, Martin, Kikuchi, \& Mazuka, 2017) but not in Dutch, Norwegian, Japanese, and German (Audibert \& Falk, 2017; Benders, 2013; Englund, 2018). The reasons underlying these cross-linguistic differences remain unclear, but we have chosen to focus on these two properties of IDS since we studied Australian English where they have been widely documented in previous studies (Burnham, et al., 2002; Kalashnikova \& Burnham, 2018; Kalashnikova, et al., 2017; Kalashnikova et al., 2018; Lam \& Kitamura, 2010, 2012; Xu, et al., 2013).

The linguistic function of IDS has been supported by findings that infants are more successful in a number of language processing tasks when presented with stimuli in IDS than in ADS. It has been found that the distinctive prosody of IDS facilitates infants' phonetic discrimination (Trainor et al., 2000), word segmentation (Thiessen, Hill, \& Saffran, 2005), and novel word-referent mapping (Graf Estes \& Hurley, 2013; Ma, Golinkoff, Houston, \& Hirsh-Pasek, 2011). Slow speaking rate and vowel hyperarticulation in IDS have been shown to promote infants' efficiency in spoken language processing (Song, Demuth, \& Morgan, 2010) and vowel discrimination (Peter, Kalashnikova, Santos, \& Burnham, 2016; Zhang et al., 2011). At the individual level, mothers' vowel hyperarticulation also has been linked to the development of their infants' speech perception (Liu, Kuhl, \& Tsao, 2003) and 
vocabulary growth (Hartman, Bernstein Ratner, \& Newman, 2017; Kalashnikova \& Burnham, 2018). Thus, IDS not only attracts infants' attention and facilitates processing of the speech signal, but it also appears to facilitate the development of their speech perception and vocabulary growth. Over and above these considerations, research has shown that the nature of IDS is shaped by infant age and their changing linguistic needs. However, the findings regarding pitch and vowel hyperarticulation modifications in IDS over development are mixed. With regards to pitch, pitch height has been shown to increase up to around 12 months (Kitamura \& Burnham, 2003; Kitamura, Thanavishuth, Burnham, \& Luksaneeyanawin, 2001) and decrease around 16 to 30 months of age (Stern, Spieker, Barnett, \& MacKain, 1983). These adjustments in pitch height across ages may be explained by different infant linguistic needs at specific ages and maternal usage of different acoustic cues in IDS to fulfill those needs. Thus, greater pitch height at six and 12 months may be used to comfort the infant or to encourage attention, while lower pitch height may be a result of attempts to direct infants' behaviour (Kitamura \& Burnham, 2003), which may be related to infants' increased attention to specific phonemes in their native language around 6 to 9 months (Hayashi, Tamekawa, \& Kiritani, 2001). On the other hand, two studies have not found changes in pitch height over development. Narayan and McDermott (2016) have not found differences in pitch height and range in infants from four to 16 months of age who were acquiring Tamil, Tagalog or Korean. Similarly, pitch was found to be stable in infants from seven to 18 months of age acquiring Australian English (Kalashnikova \& Burnham, 2018). These contradictory findings warrant further investigation of pitch features over development. With regard to vowel hyperarticulation, recent studies have found similar degrees of vowel hyperarticulation in IDS to infants from 3 to 20 months of age (E. Burnham et al., 2015; Cristia \& Seidl, 2014; Kalashnikova \& Burnham, 2018), but earlier accounts have also proposed that the degree of vowel hyperarticulation in maternal IDS increases as a function of their infants' increasing linguistic competence, in particular, expressive language skills (Bernstein Ratner, 1984). 
The effects of the linguistic needs of the audience on vowel hyperarticulation have been further demonstrated in research on distinct speech registers; vowels in speech directed to foreigners (Uther, et al., 2007) and computers (Burnham, Joeffry, \& Rice, 2010) are hyperarticulated, but not in speech directed to pets (Burnham, et al., 2002), unless that pet is a parrot (Xu, Burnham, Kitamura, \& Vollmer Conna, 2013). Aside from linguistic competence, speakers are also sensitive to listeners' linguistic processing needs. For example, vowel hyperarticulation is found in speech in noise (Castellanos, Benedi, \& Casacuberta, 1996) and speech in challenging conditions (Hazan \& Baker, 2011). Of most relevance for this study, vowel hyperarticulation has also been found in speech to adults with HL (Ferguson \& Kewley-Port, 2002, 2007; Hazan \& Baker, 2011), which further suggests that listeners' sensory abilities impact the qualities of speech addressed to them. It could be that in these speech contexts, speakers receive cues via covert or even via direct feedback from their listeners, or that speakers unconsciously adjust their speech through the process of phonetic convergence or accommodation (Pardo, 2006). Similar adjustments to vowel hyperarticulation have been observed in IDS to infants who are affected by deficits in auditory processing (infants at-risk for developmental dyslexia, Kalashnikova, Goswami, \& Burnham, 2018; 2020), or who are unable to hear their mother's speech (Lam \& Kitamura, 2012). Thus, it appears that caregivers' speech may be sensitive to their infants' ability to hear and process speech, which raises the possibility that the acoustic properties of maternal IDS to infants with HL may be different compared to IDS to infants with NH.

\section{A. Acoustic Features of Infant-directed Speech to Infants with Hearing Loss}

Research on IDS to infants with HL has focused primarily on heightened pitch and vowel hyperarticulation due to their proposed attention-getting and linguistic functions respectively. With regard to pitch, the degree to which mothers exaggerate pitch in their IDS appears to be modulated by their infants' hearing experience and not maturational factors. Mothers have been found to exaggerate pitch height (Bergeson, Miller, \& McCune, 2006) and pitch range (Miyamoto, Houston, \& Bergeson, 
2005) in speech to infants with HL between 10 and 37 months to a similar degree as to controls with NH matched by hearing age. On the other hand, pitch height and range to infants with HL were found to be greater when compared to NH controls matched by chronological age (infants' chronological age ranged from 3 to 18 months in this study, Bergeson et al., 2006). These findings suggest that mothers adjust pitch in IDS according to their infants' hearing experience and not their chronological age. Given the finding that infants with HL exhibit lower responsiveness during mother-infant interactions compared to infants with NH (Koester, 1995), mothers of infants with HL might face greater demands for attracting and maintaining their infants' attention to speech. Thus, greater pitch in IDS to infants with HL compared to infants with NH may be the result of mothers trying to attract infants' attention by increasing their pitch. This in turn can also lead to raised formant frequencies (Kalashnikova et al., 2017).

Vowel hyperarticulation has been documented in IDS to infants with HL, but this has been found to be modulated as a function of their infant's HL and level of speech processing possible through their particular hearing intervention device. For instance, mothers have been shown to hyperarticulate vowels to infants with Cochlear Implants between 11 and 27 months to a similar degree as to chronological- and hearing-age-matched controls with $\mathrm{NH}$ (Wieland, et al., 2015) and to 11month-old profoundly deaf infants prior to Cochlear Implantation compared to infants with $\mathrm{NH}$ (Kondaurova, Bergeson, \& Dilley, 2012). In addition, Wieland et al. (2015) found greater vowel dispersion in IDS than in ADS to both infants with HL and infants with NH suggesting greater vowel variability in IDS, and interestingly, this study reported greater vowel space dispersion in IDS to infants with Hearing Aids but not Cochlear Implants.

More detailed evidence on mothers' vowel production in IDS to infants with HL comes from direct measures of individual formant frequencies (F1 and F2) and vowel space dispersion. Regarding formant frequencies, Wieland et al. (2015) found higher F1 for /i/ in IDS to infants with Hearing Aids, 
and higher F2 for vowels /a/ and /i/ in IDS to infants with Cochlear Implants compared to infants with NH both chronologically and hearing-age matched. High F1 and F2 frequencies are important for vowel intelligibility and speech comprehension (Smiljanić \& Bradlow, 2009; Ferguson \& KewleyPort, 2002; 2007), so it could be that mothers compensate for their infants' HL by producing higher formant frequencies to make their vowels clearer and more intelligible.

Although cross-sectional evidence suggests that mothers adjust their IDS to infants with HL according to their hearing experience rather than chronological age (Bergeson et al., 2006; Kondaurova \& Bergeson, 2011), very little attention has been paid to the developmental adjustments in vowel and pitch production in IDS to these infants. To date, only developmental changes in pitch production have been assessed demonstrating the absence of changes in acoustic modifications over the 12-month period at three, six, and 12 months post-implantation (Kondaurova, Bergeson, \& Xu, 2013). These findings are in accordance with findings on stability of pitch height over development in infants with NH (Kalashnikova \& Burnham, 2018; Narayan \& McDermott, 2016). Given the importance of pitch for attracting infants' attention to speech and the potential linguistic role of vowel hyperarticulation, a longitudinal assessment of these features in IDS to infants with HL is required to clarify any modifications of pitch and vowel hyperarticulation in speech to infants as a function of age and hearing experience.

Before turning to the objectives of the present study, it is important to consider several factors that may underlie individual differences in maternal IDS to infants with HL, and that may account for some inconsistencies across previous studies. These factors can lead to variability in the acoustic input received and the auditory information perceived by each infant depending on the type of hearing device, configuration of HL, and the degree of HL. First, it is important to note that the nature of the acoustic input from Hearing Aid and from Cochlear Implant devices is not only significantly different from the sound conducted through a normally functioning ear but also significantly different from each 
other (Houston, Ying, Pisoni, \& Kirk, 2001). Second, infants' auditory perception varies depending on whether their HL is bilateral or unilateral. For instance, infants with unilateral HL have delayed vocabulary development compared to infants with bilateral HL (Fitzpatrick et al., 2019; Välimaa et al., 2018), and infants with bilateral HL show better sound localisation acuity and speech perception in noise (Johnston, Durieux-Smith, Angus, O’Connor, \& Fitzpatrick, 2009) and develop higher receptive and expressive vocabulary skills compared to infants with unilateral HL (Boons et al., 2012; Sarant, Harris, Bennet, \& Bant, 2014). While no studies to date have investigated the effects of bilateral vs. unilateral HL configuration on IDS components, differences in IDS qualities to these infants could be expected given the factors described above as well as other experiential factors such as different intervention approaches for infants with unilateral and bilateral HL, later age of fitting for infants with unilateral HL (Fitzpatrick, Whittingham, \& Durieux-Smith, 2014), and greater confusion among parents of infants with unilateral HL regarding effectiveness of intervention practices (Fitzpatrick et al., 2015).

Infants with HL can also be categorized according to their degree of HL regardless of its types and causes and the device used. Individual degree of HL is determined through hearing threshold testing. Based on the classification of the Joint Committee on Infant Hearing (2007), HL can be defined as mild, moderate, severe, profound or as a combination of these. Mild HL occurs when hearing thresholds are between 21 and $40 \mathrm{~dB}$. Moderate HL refers to hearing thresholds between 41 and $70 \mathrm{~dB}$ and in these cases a child will need to be fitted with Hearing Aids in order to understand normal speech. A hearing threshold between 71 and $90 \mathrm{~dB}$ is defined as severe HL and includes difficulty in understanding normal speech even with Hearing Aids. If hearing thresholds are at $91 \mathrm{~dB}$ or greater, this is defined as profound HL and in these cases, the child is unable to hear and understand a shouted voice even with Hearing Aids (Joint Committee on Infant Hearing, 2007). Additionally, HL can include a combination of these types such as cases when a child has, for example, a moderate HL in 
the low frequency plus a profound HL in the high frequency range, which would be described as moderate to profound HL.

Another important factor that must be considered in this research is the difference between chronological age and amount of post-birth hearing experience. In infants with $\mathrm{NH}$, chronological age reflects language experience, and it has been proposed that pitch and affect in mothers' IDS are modified as their infants grow older and therefore acquire more extensive language experience (Kitamura et al., 2001). In the case of infants with HL, it is unclear whether any adjustments in IDS reflect infants' chronological age or their hearing age, which captures their experience with hearing and language use. One way to assess this is by comparing IDS to infants with HL to two control groups of infants with NH: chronological age controls - infants with $\mathrm{NH}$ of the same chronological age as the infants with HL, and hearing age controls - infants with $\mathrm{NH}$ with the same amount of hearing experience (post-birth) as the infants with HL (post fitting/implantation of Hearing Aids/Cochlear Implants). Another way to assess whether the adjustment in IDS to infants with HL are the result of infants' chronological age or hearing experience is by implementing a longitudinal assessment. In this way an individual infant's growth in chronological and hearing age can be tracked, which gives a more precise measure of how these two factors influence the IDS features across development and language acquisition in these infants. Additionally, the implementation of longitudinal designs has the potential to inform the possible modifications and stability of these features across infants' development. Both methods were used in Experiments 1 and 2 reported here.

The main goal of Experiment 1 was to assess the acoustic features in IDS to infants with HL specifically focusing on infants' chronological age and hearing experience. Three groups of motherinfant dyads participated: infants with HL, infants with NH matched by chronological age, and infants with NH matched by hearing age to infants with HL. In all three groups, mothers' speech was recorded when they spoke to their infant (IDS) and when they spoke to another adult (ADS). Detailed acoustic 
analyses were conducted on mothers' productions of the three corner vowels /a, i, u/ (formant dispersion, F2-F1 distances, vowel space area, and vowel space dispersion) and utterance-level pitch (mean and variation of fundamental frequency) to compare mother's IDS to their own ADS and to compare IDS features across the three infant groups. The aim of Experiment 2 was to assess longitudinally the effects of infants' chronological age and hearing experience on vowel and pitch production in maternal IDS relative to their own ADS. This was achieved via a longitudinal assessment of the adjustments of IDS to infants with HL as a function of their increasing hearing experience across development. Maternal IDS features were assessed when infants with HL were 11- and at 18-monthsold and had eight and 15 months of hearing experience respectively. In addition, Experiment 2 examined the relation between IDS features and infants' developing lexical abilities.

\section{EXPERIMENT 1}

Previous research has indicated that infants with HL may receive exposure to IDS that is qualitatively different from the input of age- or hearing experience-matched infants with $\mathrm{NH}$ (Bergeson et al.,, 2006; Kondaurova et al., 2012; Lam \& Kitamura, 2010; Miyamoto et al., 2005). However, the exact nature and implications of these differences remain unclear. It is possible that mothers of infants with HL unconsciously produce clearer IDS by producing speech that allows easier discrimination between speech sound categories (Wieland et al., 2015). On the other hand, it is possible that the speech sound exaggeration component is absent in IDS to infants with HL, which could be a by-product of greater acoustic variability, that is more dispersed speech sounds within speech sound categories, aimed at capturing and maintaining infants' attention to speech (McMurray, Kovack-Lesh, Goodwin, \& McEchron, 2013).

This experiment assessed the effects of hearing experience on the acoustic exaggeration (hyperarticulated vowels and exaggerated pitch) and acoustic variability in IDS compared to ADS. First, with respect to the comparison between IDS and ADS, we expect to find greater acoustic 
exaggeration (hyperarticulated vowels, exaggerated pitch evident in greater pitch height, and greater pitch variation) in IDS than in ADS for all three groups of infants (Wieland et al., 2015). Between the groups, two alternate hypotheses can be entertained. First, if mothers adjust their speech due to their infants' hearing level, then we expect different IDS features in IDS to infants with HL compared to IDS to both chronological- and age-matched controls, with no difference between the two NH control groups (Kondaurova et al., 2012; Lam \& Kitamura, 2010). Second, if mothers adjust their speech due to infants' hearing experience, then we expect to find that IDS to chronological age-matched controls with NH will differ from IDS to infants with HL and hearing age-matched controls.

In order to assess variability in vowel production, we adopted three different measures: vowel space dispersion, formant dispersion, and F2-F1 distances for corner vowels (/i, a, u/). Vowel space dispersion captures the distance between a central point in the speaker's vowel space and each token of a vowel. This measure captures the overall expansion or compression of individual speakers' vowel tokens and allows the detection of fine-grained individual differences in acoustic-phonetic characteristics. Using this measure, greater vowel space dispersion indicates clearer vowels and captures a different aspect of vowels than the vowel hyperarticulation measure (Kuhl et al., 1997); it provides a measure of vowel clarity and within-category variability. Greater vowel space dispersion in IDS compared to ADS would provide evidence of vowel enhancement in IDS (Bradlow, Torretta, \& Pisoni, 1996; Smiljanic \& Bradlow, 2005; Wieland et al., 2015). Another measure of vowel variability involves computing the standard deviations separately for F1 and F2 frequencies for each of the three corner vowels /i, u, a/ for IDS and ADS (formant dispersion measure). The presence of greater standard deviation values for F1 in IDS compared to ADS would indicate less clear vowels in terms of vowel height, and greater standard deviation values for F2 in IDS compared to ADS would indicate less clear vowels in terms of the vowel backness. Previous studies using this measure have found greater vowel variability in IDS compared to ADS (Benders, 2013; Cristia \& Seidl, 2014; Englund, 2018; McMurray, Kovack-Lesh, Goodwin, \& McEchron, 2013). This greater variability has 
been shown to result in overlapping vowel categories, which are thought to complicate infants' vowel acquisition in their native language (Benders, 2013; Cristia \& Seidl, 2014; Englund, 2018; McMurray, et al., 2013). If there is greater variability in IDS than in ADS this would provide evidence for a lack of vowel enhancement in this register. We also add one more measure that captures the distances between F1 and F2 for each of the corner vowels /i, u, a/. Each of these vowels represents an extreme point in Australian English vowel space. Acoustically they are characterised by extreme F2-F1 distances: /i/ is characterised by a wide separation between F1 and F2, whereas /a/ and /u/ are characterised by very close F1 and F2 frequencies. Hence, the F2-F1 distances for these corner vowels provide an indication of the extreme locations in the F1 by F2 space for these vowels (Gerstman, 1968), which is a significant correlate of overall speech intelligibility (Bradlow et al., 1996).

\section{A. Method}

a. Participants. Sixty mother-infant dyads participated. In 20 dyads the infant had congenital HL (HL group; Mean Age = 15.09 months, standard deviation, $S D=9.06$, Age range $=7.17-35.86$, 10 female). Within this group, 11 infants had mild to moderate HL and 9 severe to profound HL, and 14 infants had bilateral HL and 6 unilateral HL (see supplementary materials for further details). Forty infants had normal hearing; 20 matched to the HL group by chronological age (N-CA group, Mean Age $=15.37$ months, $S D=8.87$, Age range $=6.90-35.86,7$ female $)$ and 20 by hearing age $(\mathrm{N}-\mathrm{HA}$ group, Mean Age $=11.68$ months, $S D=8.43$, Age range $=5.23-32.48,6$ female).

All mothers were native speakers of Australian English with NH (Mean age $=33.66, S D=$ 4.74), and all infants were raised in a monolingual context, born full-term, and not at-risk for any additional developmental disorders. Mothers' median education level was a University (bachelor) degree, and a Kruskal-Wallis $\mathrm{H}$ test showed that it did not differ across the three groups $\left(\chi^{2}(2)=2.058\right.$, $p=.357$ ). Five mother-infant dyads (2 HL, 2 NH-CA, and 1 NH-HA) were detected as outliers (hyperscores for vowel hyperarticulation were higher than three standard deviations from the mean). The 
reason for these outlier hyperarticulation scores is that the five participants only produced two or fewer tokens of each vowel in ADS, which resulted in an unusually small ADS area, not representative of the typical vowel production in ADS. These five dyads and dyads from their corresponding matched groups were excluded from analyses (15 dyads excluded in total). Thus, the final sample comprised 45 dyads, 15 with HL, 15 NH-CA, and 15 NH-HA.

b. Procedure. Mothers' speech was recorded in two situations: a play session with their infant (IDS) and a semi-structured interview with an adult experimenter (ADS). The IDS play sessions were recorded in a quiet room inside an infant laboratory or a clinic. Mothers were provided with three toys, a sheep, a shoe, and a shark, and were instructed to play with their infants naturally as they would do at home. These toys were chosen in order to elicit the target words sheep, shoe, and shark (note that ' $\mathrm{r}$ ' is non-rhotic in Australian English), and mothers were not aware that the specific vowels /a, i, u/ were the focus of this study. Mothers wore a head-mounted microphone (AudioTechnica A892) feeding into Adobe Audition CS6 software via an audio input/output device (MOTU Ultralite MK3). The ADS sessions were conducted in the same way. During this session, a female experimenter, a native speaker of Australian English, interviewed each mother about the IDS session, eliciting the same three target words. The infant was not present in the room during this session. The IDS and ADS sessions lasted between 5 and 7 minutes each.

\section{Analyses}

a. Vowel hyperarticulation. For analyses, the target words sheep, shoe and shark were identified in each IDS and ADS recording, their onset and offset were manually determined, and then each of the words was extracted. Next, the target corner vowels /a, i, u/ were extracted from each of these words (see supplementary materials for mean number of extracted vowels). PRAAT scripts were then used to obtain the values for duration, F0, F1 and F2 for each vowel. The formant values used were the mean value in $\mathrm{Hz}$ from the $40 \%$ and $80 \%$ points of each vowel's duration (Munhall, 
MacDonald, Byrne, \& Johnsrude, 2009). Mean F1 and F2 values in Hz were used to calculate vowel space area separately for IDS and ADS as a measure of vowel hyperarticulation using the following formula:

Vowel area $=$ ABS $1 / 2 \times[\mathrm{F} 1 / \mathrm{a} / \times(\mathrm{F} 2 / \mathrm{i} /-\mathrm{F} 2 / \mathrm{u} /)+\mathrm{F} 1 / \mathrm{i} / \times(\mathrm{F} 2 / \mathrm{u} /-\mathrm{F} 2 / \mathrm{a} /)+\mathrm{F} 1 / \mathrm{u} / \times(\mathrm{F} 2 / \mathrm{a} /-\mathrm{F} 2 / \mathrm{i} /)]$.

In order to ensure that any differences in vowel hyperarticulation found between the dyads with infants with $\mathrm{HL}$ and infants with $\mathrm{NH}$ were not due to additional modifications to vowel production, we also assessed the duration and pitch height for each vowel produced in ADS and IDS. (see supplementary materials for mean values for each group). As expected, univariate ANOVAs showed that vowels were longer, $F(1,88)=11.65, p=.001, \eta_{p}{ }^{2}=.117$, and higher in pitch, $F(1,88)$ $=166.59, p=.01, \eta_{p}{ }^{2}=.654$, in IDS compared to ADS. However, and critically for this study, there were no significant group differences in vowel duration and pitch for both IDS (duration: $F(2,42)=$ $1.49, p=.237, \eta_{p}^{2}=.066$; pitch: $\left.F(2,42)=.005, p=.995, \eta_{p}^{2}=.01\right)$ and ADS registers (duration: $\left.F(2,42)=.773, p=.468, \eta_{p}{ }^{2}=.036 ; F(2,42)=2.58, p=.09, \eta_{p}{ }^{2}=.109\right)$.

b. Variability in vowel production. Vowel space dispersion was calculated for both IDS and ADS by identifying the centroid of each speaker's vowel space triangle and then computing the distances of individual vowel tokens from the centroid (Bradlow et al., 1996; Wieland et al., 2015). Formant dispersion was calculated using standard deviations for F1 and F2 (Cristia \& Seidl, 2014; Englund, 2018) for each corner vowel in IDS and ADS. The measure of F2-F1 distances was calculated by subtracting F1 values form F2 values separately for /i/, /a/ and /u/ in IDS and ADS.

c. Pitch exaggeration and variability. In order to analyse pitch, IDS and ADS recordings were separated into audio segments using PRAAT software (Boersma \& Weenink, 1996). The segments were defined as a period of mother's speech not interrupted by infant's vocalisations or noises from the environment. The segments were between six and 12 seconds long (see supplementary materials for mean duration of audio segments). From these audio segments, mean fundamental frequency (F0) 
was extracted. Since pitch perception is logarithmic by nature, all F0 values were converted from $\mathrm{Hz}$ into perceptual units (Mels):

Semitone $=12 \mathrm{LOG}_{2}(\mathrm{F0})$ for pitch height.

As a measure of pitch variability, we calculated F0 standard deviations in both registers.

\section{B. Results}

\section{Hyper-Scores for vowel articulation, vowel space dispersion, and pitch}

Hyper-scores for vowel articulation (vowel triangle area), vowel space dispersion, and pitch were calculated by dividing each mother' IDS scores by her own corresponding ADS scores. This controls for individual differences by using each speaker's ADS productions as their own baseline (Kalashnikova \& Burnham, 2018); importantly, this captures the degree to which each feature is exaggerated in IDS compared to each mother's ADS. In hyper-scores, values $>1$ signify hyperarticulation - expanded vowel triangle, more dispersed vowels, heightened pitch in IDS compared to ADS; values $<1$ signify hypoarticulation - reduced vowel triangle, less dispersed vowels, reduced pitch compared to ADS; and values $=1$ signify an IDS production not different from ADS. One-sample $t$-tests were used to compare each hyper-score to the value of 1 and univariate ANOVAs with hyper-scores as the dependent variable and group (HL, NH-HA, NH-CA) as the independent variable were conducted to compare hyper-scores for vowel articulation, vowel space dispersion, and mean pitch across the three groups of infants (see Figure 1 for hyper-scores and Table I for the summary of $t$-test results). Additionally, in order to evaluate the evidence for the null and research hypothesis, Bayes factors were determined using the Jeffreys-Zellner-Siow (JZS) method, since this method is suggested to be convenient for studies with small sample sizes (Jarosz \& Wiley, 2014). In order to interpret the Bayes factors, we used the conventional cut-offs based on Jeffreys (1998), with

a Bayes factor greater than 3 representing sufficient evidence for the experimental hypothesis, and 
Bayes factor less than .33 , representing sufficient evidence to be taken for the null hypotheses. The Bayes factor with the values between approximately .33 and 3 indicate that the data are insensitive.

Table I. Results of one-sample t-test analyses (Cohen's d) comparing hyper-vowel, hyperdispersion, and hyper-pitch scores to $1(d f=14)$ in IDS to infants with HL, NH-CA, and NH-HA infants $(* * p<.001)$

\begin{tabular}{llll}
\hline Group & Hyper-vowels & Hyper-dispersion & Hyper-pitch \\
\hline HL & $.82(.22)$ & $-.13(.03)$ & $6.88^{* *}(1.75)$ \\
NH-CA & $-1.19(.31)$ & $-1.81(.48)$ & $8.82^{* *}(2)$ \\
NH-HA & $.19(.05)$ & $-1.44(.38)$ & $11.37 * *(2.5)$ \\
\hline
\end{tabular}

Additionally, linear mixed effects model (LME) was used to assess the F2-F1 distances in IDS across tokens for each corner vowel across the groups. The model was fitted using the lmer function of the lme4 package (Bates, Maechler, \& Bolker, 2013) in R (R core team, 2017) with Vowel and Group as the independent variables, Vowel * Group interaction, and random intercepts for Participants, and F2-F1 distance as the dependent variable. The significance of the model was assessed using ANOVAs with Satterthwaite's method using the anova function of the lmerTest package (Kuznetsova, Brockhoff, \& Christensen, 2017) and the Bayes factor was determined using the function bayesfactor_models from the R package bayestestR (Makowski et al., 2020).
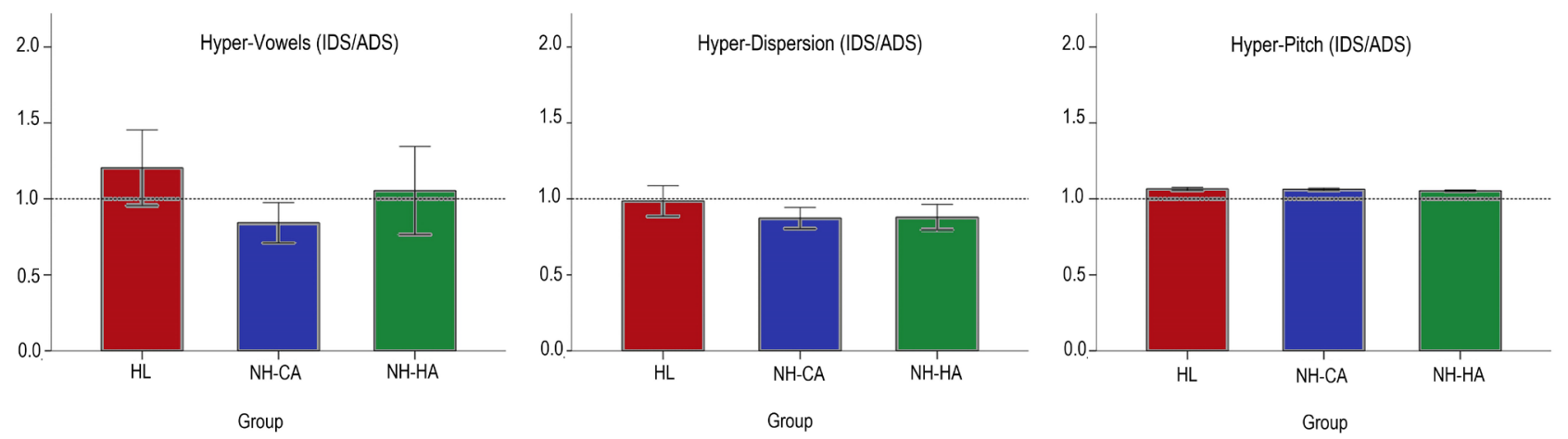

Figure 1. Hyper-scores for vowel articulation, vowel space dispersion, and mean pitch for infants with HL, NH-CA and NH-HA infants (error bars represent standard error of mean, SEM). 


\section{Vowel production in IDS: exaggeration}

a. Vowel hyperarticulation. The one-sample $t$-tests showed that in IDS to all three groups of infants, mothers did not expand or reduce their vowel space compared to their ADS. The univariate ANOVA demonstrated no significant group effects $\left(F(2,42)=.608, p=.549, \eta_{p}{ }^{2}=.028\right)^{\mathrm{c}}($ see supplementary materials for vowel triangles). The Bayes factor obtained for this analysis $(\mathrm{BF}=.038)$ supports the null hypothesis that there is no significant group difference in the vowel hyperarticulation in IDS. While there are mixed findings in research on IDS to infants with HL, these results are in line with studies that have not found consistent differences in vowel hyperarticulation (Wieland et al., 2015) as a function of infants' HL.

\section{Vowel production in IDS: variability}

a. Vowel space dispersion. As can be seen in Table I, the one-sample $t$-tests indicated that mothers produced vowels with a similar amount of dispersion in IDS to all three groups of infants as compared to ADS. The univariate ANOVA showed no significant difference across the three groups $\left(F(2,42)=.550, p=.581, \eta_{p}^{2}=.026\right)$ with the Bayes factor $(\mathrm{BF}=.036)$ showing strong evidence for the null hypothesis.

b. Formant dispersion. To assess the variability across the tokens for each corner vowel, measures of F1 and F2 standard deviations in IDS and ADS were used. Two 2 (speech register: IDS,

${ }^{\mathrm{c}}$ Given the wide age range included in this study, additional Analyses of Covariance were conducted with hyper-scores for vowel area, dispersion, and pitch as dependent variables, group as the independent variable and age in months as the covariate. Results yielded identical patterns to the analyses reported above. That is, after controlling for the effect of the age on hyper-scores, there was no significant difference across the three groups in vowel space areas $\left(F(2,41)=.586, p=.561, \eta_{p}{ }^{2}\right.$ $=.028)$, vowel space dispersion $\left(F(2,41)=.508, p=.606, \eta_{p}^{2}=.024\right)$, and in pitch $(F(2,42)=1.791$, $\left.p=.180, \eta_{p}^{2}=.080\right)$. Importantly, there were no significant effects of age on vowel area $(F(2,41)=$ $\left..624, p=.434, \eta_{p}^{2}=.015\right)$, vowel space dispersion $\left(F(2,41)=.154, p=.697, \eta_{p}^{2}=.004\right)$ and, but there was a significant effect of age on $\operatorname{pitch}\left(F(2,41)=8.639, p=.005, \eta_{p}{ }^{2}=.174\right)$. 
ADS) $\times 3$ (Group: HL, NH-CA and NH-HA) mixed-measures ANOVAs were conducted separately for each vowel /a, i, u/. We present the results of the ANOVAs in Table II.

These results indicate that formant dispersion for /a/ (IDS: $M=89.90$, standard error, $S E=$ 4.04; ADS: $M=77.05, S E=4.04 ; p=.03)$ and /i/ (IDS: $M=299.19, S E=12.64$; ADS: $M=243.86$, $S E=14.87 ; p=.01)$ but not for $/ \mathrm{u} /$ was greater in IDS compared to ADS, suggesting more variability in IDS than ADS but with no difference across the three groups of infants.

Table II. Analyses of formant dispersion in IDS and ADS using 2 (Speech Register: IDS, ADS) $x 3$ (Group: HL, NH-CA and NH-HA) mixed-measures ANOVAs for vowels /a, $i, u /(N=45)$

\begin{tabular}{|c|c|c|c|c|c|c|c|c|c|c|c|c|}
\hline & \multicolumn{4}{|c|}{$\begin{array}{l}\text { Register (IDS vs. } \\
\text { ADS) }(\mathrm{df}=1 \\
42)\end{array}$} & \multicolumn{4}{|c|}{$\begin{array}{l}\text { Group (HL vs. } \\
\text { NH-CA vs. NH- } \\
\text { HA) }(\mathrm{df}=2,42)\end{array}$} & \multicolumn{4}{|c|}{$\begin{array}{l}\text { Register x Group } \\
(\mathrm{df}=2,42)\end{array}$} \\
\hline & $F$ & $p$ & $\eta_{p}^{2}$ & $B F$ & $F$ & $p$ & $\eta_{p}^{2}$ & $B F$ & $F$ & $p$ & $\eta_{p}^{2}$ & $B F$ \\
\hline /a/ F1 & 4.85 & .03 & .10 & 2.12 & .58 & .56 & .03 & .15 & .06 & .94 & .01 & .05 \\
\hline F2 & 3.32 & .08 & .07 & 1.17 & .15 & .86 & .01 & .10 & 1.21 & .31 & .05 & .05 \\
\hline /i/ F1 & 1.16 & .29 & .03 & .36 & 2.02 & .15 & .09 & .68 & .21 & .81 & .01 & .04 \\
\hline F2 & 8.12 & .01 & .16 & 6.49 & 2.84 & .07 & .03 & .73 & .06 & .94 & .01 & .94 \\
\hline$/ \mathrm{u} / \mathrm{F} 1$ & 3.57 & .07 & .08 & .65 & .63 & .54 & .03 & .20 & .98 & .38 & .04 & .03 \\
\hline F2 & .03 & .86 & .01 & .22 & .21 & .81 & .01 & .12 & .58 & .57 & .03 & .01 \\
\hline
\end{tabular}

c. F2 - F1 distances. The LME results demonstrated a significant main effect of Vowel $(F(2$, $1352.98)=285.91, p<.001)$, but there was no significant main effect of Group $(F(2,42.88)=.607, p$ $=.55)$, and no significant Vowel by Group interaction $(F(2,1352.95)=.862, p=.486)$ with Bayes factor $(\mathrm{BF}=1.77)$ suggesting anecdotal evidence against the null hypothesis.

\section{Pitch production in IDS: exaggeration}

a. Pitch height. Mothers significantly exaggerated their pitch height in IDS compared to ADS to all three groups of infants (see Table I). The univariate ANOVA of pitch hyper-scores showed no significant group effect $\left(F(2,42)=.783, p=.464, \eta_{p}^{2}=.036, \mathrm{BF}=0.45\right)$; thus mothers exaggerated 
pitch in IDS compared to ADS to an equivalent degree in all three groups of infants. This is in line with studies that have found no consistent differences in pitch as a function of infants' HL (Bergeson et al., 2006; Lam \& Kitamura, 2010; Miyamoto et al., 2005).

\section{Pitch production in IDS: variability}

a. Pitch variability. In order to compare pitch variability, a repeated measures ANOVA was conducted with Speech register (IDS, ADS) as a repeated factor and Group as a between-subjects factor. The results demonstrated a main effect of Speech register $\left(F(1,54)=52.099, p=.000, \eta_{p}{ }^{2}=\right.$ $.491, \mathrm{BF}>100)$; pitch variation was greater in IDS $(M=35.486, S D=15.524)$ compared to ADS $(M$ $=17.983, S D=8.949)$. There was no significant difference between groups $(F(2,54)=1.240, p=$ $\left..297, \eta_{p}^{2}=.044, \mathrm{BF}=0.3\right)$, and no significant register by group interaction $(F(2,54)=.162, p=.851$, $\left.\eta_{p}^{2}=.006, \mathrm{BF}>100\right)$

\section{Discussion}

Experiment 1 compared the acoustic features of IDS to infants with HL and to infants with NH matched by chronological or by hearing age. The findings indicated no significant group differences in vowel or pitch exaggeration, which is in line with a number of previous studies (Bergeson et al., 2006; Lam \& Kitamura, 2010; Miyamoto et al., 2005; Wieland et al., 2015). On the other hand, the findings suggest greater variability in vowel production in IDS compared to ADS, regardless of infants' hearing status. Although previous studies have demonstrated that variability across phonetic dimensions may hinder category learning by making vowels less clear and more difficult to learn (Cristia \& Seidl, 2014; Englund, 2018; McMurray et al., 2013), it could be that this variability would maintain infants' attention to speech for a greater period of time, which may reflect infants' linguistic needs, and parents' sensitivity to these needs at this stage in infants' development. In order to further 
disentangle the effects of infants' chronological age from their hearing experience on maternal IDS, a longitudinal assessment was conducted in Experiment 2.

\section{EXPERIMENT 2}

Experiment 2 aimed to longitudinally assess the effects of infant age and changing linguistic needs on vowel and pitch production in IDS as well as the relation between these IDS features and infants' developing lexical abilities. The longitudinal approach allowed measurement of individual infant's gain in both chronological and hearing age, providing an accurate picture of age effects on IDS properties across development and language acquisition in infants with HL.

This experiment followed the sub-set of infants with HL from Experiment 1 by re-assessing the acoustic expansion and variability properties if their mothers' IDS approximately 6 months later when their chronological age was around 18 months. Infants' receptive and expressive vocabulary size at this age were also assessed. The age of 18 months was chosen for this follow-up given it marks a significant increase in infants' expressive vocabulary and the speed and efficiency of familiar word recognition (Fernald, 2000; Fernald, Perfors, \& Marchman, 2006). In other words, at this age infants get better at recognising and interpreting the same word in more diverse and challenging contexts.

On the basis of possible linguistic needs that infants may have at the two ages in this longitudinal experiment (approximately 11 and 18 months), two alternative predictions were proposed separately for pitch, vowel exaggeration, and vowel variability. With regard to pitch, if at both ages infants' linguistic needs consist of learning speech sounds as indexed by paying more attention to the speech sound categories, then we expect a similar degree of exaggeration and variability in pitch production at both ages (Kondaurova et al., 2013). On the other hand, if as a result of acquiring greater hearing experience infants already have sufficient knowledge of speech sounds, we expect less exaggeration in pitch production at the older age (Lam \& Kitamura, 2010). 
With regard to vowel hyperarticulation, if as a result of greater hearing experience, older infants need clearer phonetic categories, we expect vowel hyperarticulation to be present at the older age (Wieland et al., 2015). If infants at both ages have similar needs for learning speech sounds, then we expect to observe stability in vowel variability across age. On the other hand, if at the older age, infants would benefit from greater enhancement of vowel categories in order to learn words, then we expect the variability in terms of formant dispersion to decrease, while vowel space dispersion and distances to increase for $/ \mathrm{i} /$ and $/ \mathrm{u} /$ and to decrease for /a/. Finally, if IDS serves a linguistic function evident in the adjustment in vowel production, then we expect the measures of vowel production to be related to infants' vocabulary size (Kalashnikova \& Burnham, 2018).

\section{A. Method}

a. Participants. Eleven infants with HL and their mothers returned for a second experimental session approximately six months after taking part in Experiment 1. For this subset, infants' mean age at the first session was 10.52 months $(S D=2.18$, Age range $=8.02-14.6)$ and 17.64 months $(S D=$ 4.38, Age range $=11.77-27.94$ ) at the second session (supplementary materials for further details).

b. Procedure. Only IDS was recorded in Session 2. In order to assess the exaggeration of IDS components at both ages to the same individual baseline, ADS recordings from Experiment 1 were used. Procedures for IDS recording and analyses were the same as in Experiment 1 (see supplementary materials for details about the vowels and segments extracted for the vowel hyperarticulation and pitch height analyses). In order to ensure that any differences in vowel hyperarticulation found between the ages were not due to additional modifications to vowel production, we also assessed the duration and pitch height for each vowel produced at younger and older age. Mean values for each age are displayed in supplementary materials. Critically for this study, there were no significant differences between ages in vowel duration and pitch (duration: $F(1,17)=3.67, p=.07, \eta_{p}^{2}=.177$; pitch: $F(1,17)=.193$, $\left.p=.666, \eta_{p}^{2}=.01\right)$ 
c. Vocabulary size. During the second session, infants' caregivers completed the OZI: Australian English Communicative Development Inventory (Kalashnikova, Schwarz, \& Burnham, 2016), which is the Australian English adaptation of the MacArthur-Bates Communicative Development Inventories (MCDI, Fenson et al., 1993). Caregivers were required to select the words that their child was able to understand (receptive vocabulary) and understand and say (expressive vocabulary).

\section{B. Results}

First, the acoustic features in IDS to infants with HL were assessed. Here, the same analysis procedures were followed as in Experiment 1 with an addition linear mixed effects models (LMEs) used to compare IDS features across development. The models were fitted using the lmer function of the lme4 package (Bates, Maechler, \& Bolker, 2013) in R (R core team, 2017). Three models were fitted, with Age as the independent variable, random intercepts for Participants, and the following dependent variables: vowel hyperarticulation (Model 1), vowel dispersion (Model 2), and pitch height (Model 3).

To assess the variability in IDS across tokens for each corner vowel between the two ages, three additional LME models were fitted. These models were fitted with F1 (Model 4), F2 (Model 5) and F2-F1 distance (Model 6) as the dependent variable, Age and Vowel as independent factors, and random intercept for Participants. Pitch variability was assessed fitting the model (Model 7) with Pitch Variability as the dependent variable, Age as the independent variable, and random intercept for Participants.

The significance of each model was assessed using ANOVAs with Satterthwaite's method using the anova function of the lmerTest package (Kuznetsova et al., 2017). In the cases where the models revealed significant effects, post-hoc analyses were conducted using the Tukey test from the emmeans package (Lenth, Singmann, Love, Buerkner, \& Herve, 2019). Additionally, Bayes factors 
were determined using the function bayesfactor_models from the R package bayestestR (Makowski et al., 2020).

The ANOVA results are presented in the text, and detailed model output summaries for significant models can be found in the supplementary materials.

\section{Vowel production in IDS: exaggeration}

a. Vowel hyperarticulation. One-sample $t$-tests showed that at both ages, mothers did not expand or reduce their vowel space compared to their ADS (Age 1:t(9)=.02, $p=.99$, Cohen's $d=$ .01 ; Age 2: $t(9)=.83, p=.43$, Cohen's $d=.26$ ) (see Figure 2 for hyper-scores and supplementary materials for vowel triangles). The fitted model (Model 1) was not significant $(F(1,10)=1.56, p=$ $.24, \mathrm{BF}=1.12)$ suggesting no difference in the degree of mothers' vowel hyperarticulation at each age.
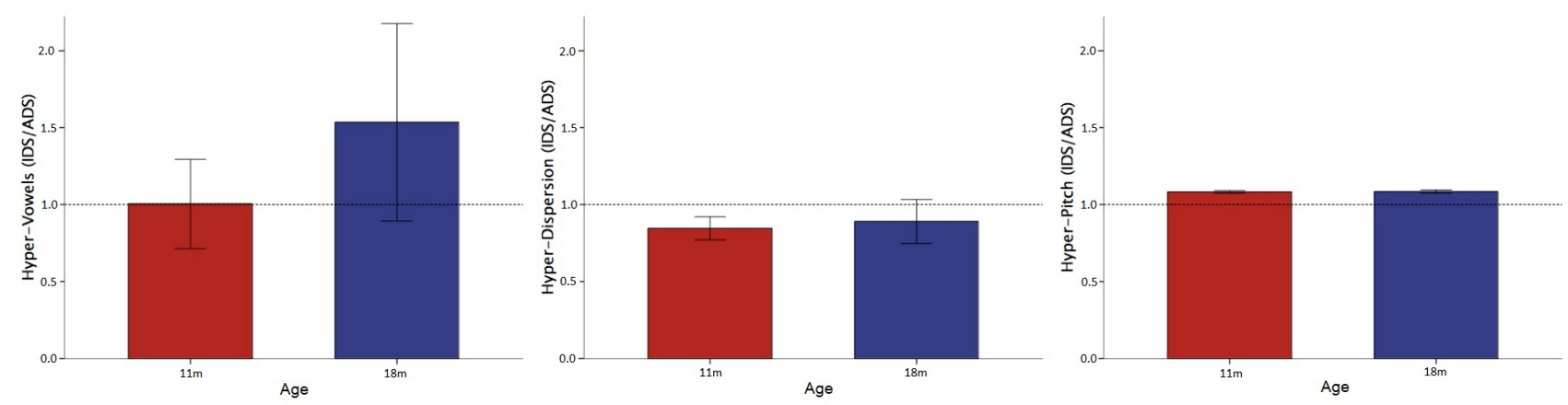

Figure 2. Hyper-scores for vowel articulation, vowel space dispersion, and mean pitch for infants with HL at 11 and 18 months (error bars represent SEM).

\section{Vowel production in IDS: variability}

a. Vowel space dispersion. One-sample $t$-tests demonstrated that at 11 months, mothers produced vowels with less dispersion in IDS than in $\operatorname{ADS}(t(8)=-2.48, p=.04$, Cohen's $d=.82)$, and with a similar amount of dispersion in IDS as in ADS at 18 months $(t(8)=-.98, p=.36$, Cohen's $d=$ .32) (see Figure 2 for hyper-scores). The fitted model (Model 2 - Vowel space dispersion) was not 
significant $(F(1,10)=1.05, p=.33, \mathrm{BF}=.13)$ suggesting that the amount of vowel space dispersion in IDS compared to ADS did not differ between the ages.

b. F1 dispersion. The LME results for F1 demonstrated that there was no significant main effect of Vowel $(F(2,72)=1.63, p=.20, \mathrm{BF}=37.03), \operatorname{Age}(F(1,72)=2.26, p=.14, \mathrm{BF}=7.26)$ and no significant Age by Vowel interaction $(F(2,72)=.76, p=.47, \mathrm{BF}>100)$.

c. F2 dispersion. The fitted LME model for F2 (Model 5) revealed a significant main effect of Vowel $(F(2,60)=31.06, p<.001, \mathrm{BF}>100)$. There was no significant main effect of Age $(F(1,60)$ $=.49, p=.49, \mathrm{BF}=8.36)$ and no significant Age by Vowel interaction $(F(2,60)=1.23, p=.30, \mathrm{BF}>$ 100). The post hoc Tukey test demonstrated greater variability in the production of F2 for vowel /i/ ( $M$ $=310, S E=18.4)$ compared to the vowels $/ \mathrm{a} /(M=131, S E=18.4), p<.001$, and $/ \mathrm{u} /(M=202, S E=$ $18.4), p<.001$. Also, there was greater variability in the production of $\mathrm{F} 2$ for the vowel $/ \mathrm{u} / \mathrm{compared}$ to the vowel $/ \mathrm{a} /, p=.01$.

d. F2 - F1 distances. The LME results demonstrated that there was a significant main effect of Vowel $(F(2,581.41)=122.78, p<.001, \mathrm{BF}>100)$, but no significant main effect of Age $(F(1,586.68)$ $=.77, p=.38, \mathrm{BF}=21.86)$, and no significant Age by Vowel interaction $(F(2,581.99)=.55, p=.58$, $\mathrm{BF}>100)$. The post hoc Tukey tests showed greater distances between F1 and F2 for vowel /i/ $(M=$ $1167, S E=24.6)$ compared to both vowels $/ \mathrm{a} /(M=800, S E=22.4, p<.001)$, and $/ \mathrm{u} /(\mathrm{M}=967, \mathrm{SE}=$ 23.9, $p<.001)$. Also, there were greater distances between F1 and F2 for vowel /u/ compared to /a/, $p$ $<.001$.

\section{Pitch production in IDS: exaggeration}

a. Pitch height. The $t$-tests showed that at both ages, mothers significantly exaggerated pitch in IDS compared to ADS (Age 1: $t(10)=9.31, p<.001$, Cohen's $d=2.67$; Age 2: $t(10)=8.28, p<$ 001, Cohen's $d=2.67$ ) (see Figure 2 for hyper-scores). The results of the LME model (Model 3 - pitch 
height) demonstrated no significant differences in maternal degree of pitch exaggeration in their IDS at the two ages $(F(1,20)=.03, p=.87, \mathrm{BF}=.01)$.

\section{Pitch production in IDS: variability}

a. Pitch variability. The fitted LME model for pitch variability (Model 7) failed to reach significance $(F(1,10)=2.24, p=.17, \mathrm{BF}=10.2)$, suggesting that there was a similar amount in pitch variability across age.

\section{Summary}

These results show that there was no exaggeration in vowel production in IDS to infants with HL either at 11- or 18-months, and no significant difference between the two ages. The vowel space dispersion measure showed that IDS to infants around 11 months was less variable in relation to ADS, while at 18 months there was a similar degree of variability in IDS compared to ADS. The second measure of vowel variability, formant dispersion, demonstrated greater variability in the production of F2 for vowel /i/ compared to vowels /a/ and /u/ and greater variability for vowel /u/ compared to vowel /a/ with no difference in these measures at 11- and 18-months of age. The third measure of vowel variability, F2-F1 distances, demonstrated wider distances between F1 and F2 for vowels /i/ compared to vowels $/ \mathrm{a} /$ and $/ \mathrm{u} /$ and greater distances for vowel $/ \mathrm{u} /$ compared to vowel $/ \mathrm{a} /$ with no difference between the younger and older age.

With regard to pitch, these results indicate that mothers significantly exaggerated pitch height in IDS compared to ADS with no difference between the two ages. In addition, these results suggest that there was a similar degree of variability in pitch production in IDS to infants with HL at both 11and 18-months of age.

\section{IDS features and vocabulary size}


Next, correlational analyses (with infants' chronological age partialled out) were conducted between infants' receptive and expressive vocabulary sizes (Receptive: $M=242.82, S D=174.06$; Expressive: $M=60.64, S D=105.54)$, and all the IDS measures collected at the two ages: vowel hyperarticulation, vowel space dispersion, hyper-pitch, distances between F1 and F2 for vowels /a/, /i/, and $/ \mathrm{u} /$.

The correlational analyses of IDS features at 11 months and vocabulary scores of infants with HL at 18 months showed no significant correlations. Table III presents correlations of IDS features at 18 months and vocabulary scores. As can be seen, at 18 months there was a positive correlation between F2-F1 distances for vowel $/ \mathrm{i} /$ and receptive vocabulary size $(r=.66, n=11, p=.04)$. In addition, there was a significant positive correlation between F2-F1 distances for the vowel /u/ at 18 months and receptive vocabulary size $(r=.64, n=11, p=.01)$. Moreover, there was a positive correlation between maternal degree of vowel hyperarticulation at 18 months and expressive vocabulary size at this age $(r=.64, n=11, p=.01)$. These results suggest that infants with HL whose mothers produced vowels /i/ and /u/ with wider separation of F1 and F2 at 18 months had larger receptive vocabulary sizes at this age. Additionally, these results indicate that infants with HL whose mothers produced IDS with more expanded vowels at 18 months had larger expressive vocabulary sizes at this age.

Table III. Pearson correlations (r) of IDS features at 18 months and infants' with HL receptive and expressive vocabulary scores at 18 months

\begin{tabular}{lllllll}
\hline & $\begin{array}{l}\text { Hyper- } \\
\text { vowels }\end{array}$ & $\begin{array}{l}\text { Hyper- } \\
\text { dispersion }\end{array}$ & $\begin{array}{l}\text { Hyper } \\
\text {-pitch }\end{array}$ & F2-F1/a/ & F2-F1/i/ & F2-F1/u/ \\
\hline Receptive & .50 & .54 & -.19 & .26 & $.66^{*}$ & $.64^{*}$ \\
Expressive & $.81^{* *}$ & -.06 & .43 & -.19 & .16 & .11 \\
\hline
\end{tabular}




\section{Individual differences in hearing configuration}

As described in the Introduction, hearing configuration is a factor that can impact language development in infants with HL, but it has never been investigated in relation to IDS. Our sample in Experiment 1 included 5 infants with unilateral and 10 infants with bilateral HL, so we conducted additional exploratory analyses comparing the acoustic components of IDS, which are reported in the supplementary materials. One-sample $t$-tests were used to compare vowel articulation and pitch hyperscores to 1, and two separate univariate ANOVAs with hyper-scores as dependent variables and group as the independent variable were conducted to assess the effects of the hearing configuration on the hyper-scores. The results showed that mothers did not expand or reduce their vowel space when addressing infants with bilateral HL $(t(9)=2.21, p=.055$, Cohen's $d=.70)$, but hypoarticulated vowels in IDS to infants with unilateral HL $(t(4)=-3.53, p=.02$, Cohen's $d=1.58)$. The degree of vowel hyperarticulation was significantly greater in IDS to infants with bilateral $\mathrm{HL}(M=1.62, S D=.89)$ compared to infants with unilateral HL $\left.(M=0.37, S D=.40), F(1,13)=8.712, p=.011, \eta_{p}{ }^{2}=.401\right)$. On the contrary, the two groups did not differ in the degree of pitch exaggeration $(F(1,13)=.470, p$ $\left.=.505, \eta_{p}^{2}=.035\right)$. The $t$-tests indicated that mothers hyper-articulated pitch in IDS to both groups of infants (Bilateral: $t(9)=4.78, p=.01$, Cohen's $d=2.67$; Unilateral: $t(4)=5.42, p=.01$, Cohen's $d=$ 2.67).

These analyses suggest that while there was vowel hyperarticulation in IDS to infants with bilateral HL, vowels in IDS to infants with unilateral HL were hypoarticulated. This could be due to different intervention approaches for infants with unilateral HL, which range from no treatment and regular monitoring, to the fitting of Hearing Aids and Bone Implant systems (Fitzpatrick et al., 2014). Also, it could be that later and more challenging fitting for infants with unilateral HL (Fitzpatrick et al., 2014) and different parental attitudes towards intervention (Fitzpatrick et al., 2015) could affect their IDS to these infants. To date, no previous studies have compared the properties of IDS to these 
two groups. However, this finding is not entirely surprising when viewed in the context of research showing that infants with unilateral HL have delayed vocabulary development and poorer auditory and language outcomes in comparison to infants with bilateral HL (Fitzpatrick et al., 2019; Välimaa et al., 2018).

\section{GENERAL DISCUSSION}

Experiment 1 assessed the acoustic features in IDS to infants with HL compared to IDS to infants with $\mathrm{NH}$ matched by either chronological or hearing age. While there were no group differences in vowel hyperarticulation and vowel space dispersion, there were differences in the production of individual vowels. With regard to pitch production, the results of Experiment 1 demonstrated heightened pitch and greater pitch variability in IDS compared to ADS with no significant group differences.

The longitudinal investigation in Experiment 2 assessed adjustments in IDS to infants with HL as a function of increasing chronological age and increasing hearing experience across development. Experiment 2 also assessed the relation between IDS features and infants' developing lexical abilities. The results showed stability in individual vowel production, pitch height, and pitch variability across development. On the other hand, the vowel space dispersion measure showed that IDS to infants at 11 months was less variable in relation to ADS, while at 18 months there was a similar degree of variability in IDS compared to ADS. Additionally, a positive relationship was found between formant distances in mothers' speech and infants' current receptive vocabulary size, as well as between vowel hyperarticulation and infants' expressive vocabulary, thus extending previous findings on this relationship for NH infants (Hartman et al., 2017; Kalashnikova \& Burnham, 2018).

The results show that there is no vowel hyperarticulation in IDS to infants with HL at either the younger or the older age. This is consistent with other studies that failed to show vowel hyperarticulation in IDS to infants with HL (Lam \& Kitamura, 2010). However, this does not 
necessarily mean that mothers do not exaggerate vowels in IDS to infants with HL; it could be that the presence of vowel variability observed in our study masked vowel hyperarticulation, and to obviate this possibility, we assessed vowel formants. Indeed, we found differences between IDS to infants with $\mathrm{HL}$ and to infants with $\mathrm{NH}$ in the production of individual vowels suggesting that maternal production of corner vowels /a, i, u/ is affected by infants' HL. Previous studies have shown that infants with HL perform more poorly than NH infants in discriminating various vowel contrasts (Eisenberg, Martinez, \& Boothroyd, 2004; Martinez, Eisenberg, Boothroyd, \& Visser-Dumont, 2008). Thus, it would appear that mothers unconsciously adjust their vowel production to the level of their infants' linguistic competence. This is in line with studies that have demonstrated that maternal speech clarity manifested in the degree of vowel hyperarticulation is modulated by infant response to the mother (Kalashnikova et al., 2018, 2020; Lam \& Kitamura, 2010, 2012).

The present results suggest that the clarity of vowel production in mothers' IDS may be affected by HL in infants. This can have important implications for language acquisition in this population given findings of the important role of speech clarity on infants' developing linguistic skills. First, it has been demonstrated that the degree of vowel hyperarticulation in IDS to six to eight and 10- to 12month-old infants is related to infants' speech perception performance (Liu et al., 2003). Thus, not hearing clear vowels may hinder speech perception in infants with HL. Since better speech perception early in life has been found to predict later language skills (Benasich \& Tallal, 2002; Molfese \& Molfese, 1985; Molfese, 2000; Tsao, Liu, \& Kuhl, 2004), this relation may be extended further to suggest that decreased clarity in maternal speech may affect different aspects of language acquisition. Thus, future studies may assess the relationship between mothers' IDS features and infants' performance on speech perception tasks. In line with findings regarding vowel hyperarticulation and infants' expressive vocabulary growth (Kalashnikova \& Burnham, 2018), our results also demonstrate a relationship between vowel hyperarticulation and infants' expressive vocabulary extending these findings to infants with HL; infants with larger expressive vocabularies at 18 months have mothers 
who produce more expanded vowel space at this age. Moreover, the results here also demonstrate a relation between formant separation and infants' receptive vocabulary, with larger receptive vocabularies in infants whose mothers produce clearer vowels via greater inter-formant distances. These findings support the argument that receiving speech input with less clear vowel production - as in the case of infants with HL - may hinder infants' receptive and expressive vocabularies. Thus, it is possible that while mothers produce less clear vowels in IDS to infants with HL, they use other cues that positively affect infants' vocabularies. This is the first study to demonstrate the relationship between exaggerated vowel production in IDS to infants with HL and their receptive and expressive vocabulary skills at 18 months of age. This suggests that hearing clearer vowels results in better language outcomes for infants with HL, specifically at 18 months of age, an age at which infants with NH enter the vocabulary spurt (Bloom, 1973).

Heightened pitch level and greater pitch variability remain stable across development. Given that infants with HL display reduced attention to speech (Houston, Pisoni, Kirk, Ying, \& Miyamoto 2003), receiving speech input with exaggerated pitch features may be beneficial in attracting infants' attention to the speech stream. Indeed, research with $\mathrm{NH}$ infants has demonstrated the benefits of heightened pitch in IDS in attracting and maintaining infants' attention to the speech input (Cooper \& Aslin, 1990; Fernald \& Simon, 1984). Thus, it is possible that mothers of infants with HL exaggerate pitch in IDS in response to their infants' greater need for speech properties that preserve their attention to the speech input.

Finally, the results of our exploratory analyses demonstrated that vowel hyperarticulation was moderated by the nature of the HL: mothers hypo-articulated vowels in IDS to infants with unilateral HL and hyper-articulated vowels in IDS to infants with bilateral HL. Although previous studies have shown that infants with unilateral HL have delayed vocabulary development and poorer auditory processing and language outcomes in comparison than infants with bilateral HL (Fitzpatrick et al., 
2019; Välimaa et al., 2018), this is the first study to demonstrate that differences in mothers' vowel hyperarticulation in IDS may be a response to different configurations of infants' HL.

\section{A. Vowel hyperarticulation in IDS}

It is noteworthy that the results of this study revealed no evidence of vowel hyperarticulation in IDS to infants with $\mathrm{NH}$, which accords with studies that suggest that this adjustment is not invariably manifested in all cases (Benders, 2013; Dodane \& Al-Tamimi, 2007; Englund \& Behne, 2005; Englund, 2018; Martin et al. 2015; Wong \& Ng, 2018), but is contrary to studies reporting significant vowel hyperarticulation in IDS (Adriaans \& Swingley, 2017; Cristia \& Seidl, 2014; Kuhl et al., 1997; Tang, Xu Rattanasone, Yuen, \& Demuth 2017; Uther et al., 2007), including findings for Australian English (Burnham et al., 2002; Kalashnikova \& Burnham, 2018; Kalashnikova et al., 2017; Kalashnikova et al., 2018; Lam \& Kitamura, 2010, 2012; Xu et al., 2013). This could be due to differences in ages at which vowel hyperarticulation was measured in this and in previous studies. Moreover, it is quite possible that the wide age range in this experiment, necessitated by the availability of infants with HL and the requirement of yoking the age of infants in both the NH-CA and NH-HA control groups to the age and hearing experience of the infants with HL served to mix ages at which vowel hyperarticulation is strong and weaker or non-existent.

\section{B. Variability in IDS}

This study has raised important theoretical and practical issues concerning the role of variability in vowel production in IDS. Previous studies that have demonstrated greater variability in vowel production in IDS than in ADS (Benders, 2013; Cristia \& Seidl, 2014; Englund, 2018; McMurray et al., 2013) have also tended to assert the detrimental effect of this variability to infants' vowel acquisition due to greater overlap between vowel categories. However, this contrasts the findings that vowel hyperarticulation results in clear vowel categories that facilitate infants' speech perception and vocabulary development (Liu et al., 2003; Kalashnikova \& Burnham, 2018). Although 
previous studies have demonstrated that the presence of variability may facilitate the acquisition of a number of early skills such as visual category learning (Mather \& Plunkett, 2011), speech segmentation (Eaves, Feldman, Griffiths, \& Shafto, 2016), and word learning (Galle, Apfelbaum, \& McMurray, 2015; Graf Estes \& Hurley, 2013; Rost \& McMurray, 2009), it should be noted that different types of variability may play different roles in language acquisition. With respect to speech input, there are two main sources of variability: variability along specific phonetic dimensions (formants in this study), and variability in non-phonetic information (pitch in this study) (Rost \& McMurray, 2009). Our finding regarding greater variability in IDS compared to ADS is consistent with other studies demonstrating that greater variability in IDS may potentially hinder category learning (Cristia \& Seidl, 2010; Englund, 2018; McMurray et al., 2013). On the other hand, our findings demonstrate the presence of greater pitch variation in IDS compared to ADS. This finding is important since previous studies demonstrated that variability in non-phonetic information such as pitch enhances attention to speech and supports the speech segmentation and to some extent vowel learning (Trainor, Austin, \& Desjardins, 2000). Therefore, our findings confirm that infants are exposed to IDS that may play an important role in their language acquisition. However, it is still unknown how IDS components interact with each other and how they relate to infants' changing linguistic needs. Nevertheless, our study suggests that a possible reason for the absence of vowel exaggeration in IDS is mothers' simultaneous increase in variability in their speech. This would make vowels less clear and more difficult to learn, but it would keep their infants' attention for longer, which may be what these infants need at this specific point in development.

How can these apparently conflicting findings be reconciled? One approach - taken in this study - is comprehensive investigation by employing a battery of different measures of vowel production. This allows for a more precise delineation of between- and within-category variability in IDS. Another possibility is to assess variability in vowel production across infant development. Thus, it is possible that increasing linguistic competence results in a concurrent increase in vowel clarity, 
and that mothers thus adjust their IDS according to infants' linguistic needs for vowel clarity in order to build their vocabularies. This suggests the importance of considering individual infants' current stage of language acquisition. Additionally, future studies should directly investigate the role of variability in vowel production on infant on-line speech processing to understand how this feature is related to infants' linguistic competence.

As can be seen, combining measures of vowel production informed our understanding of the vowel properties in IDS. This approach can be further expanded in future research by the inclusion of a wider set of vowel and consonant categories. This study analysed the production patterns of three corner vowels that occurred in three target words, which could limit our ability to detect all the acoustic adjustments that may be present in IDS to infants with HL. This approach, however, had the benefit of allowing us to strictly control for the phonetic context in which the target vowels occurred and to partially control for the content of all the interactions (i.e., even though the interactions were not structured, all mothers spoke about similar topics with their infants). Furthermore, it allows for a direct comparison between the present findings and previous studies that used the same technique for eliciting the production of the three corner vowels in IDS and ADS (Andruski \& Kuhl, 1996, Burnham et al., 2002; Kalashnikova et al., 2017; Kalashnikova \& Burnham, 2018; Kalashnikova et al., 2018; Kitamura \& Lam, 2009; Knoll \& Uther, 2004; Lam \& Kitamura, 2012; Leong, Kalashnikova, Burnham, \& Goswami, 2014; Xu et al., 2013).

The current study investigated how mothers adjust their IDS to infants with HL. While we were able to control for infants' hearing experience by including a hearing age-matched control group, we were unable to control for potential individual differences in residual hearing in the group of infants with HL and their hearing ability at the time of testing. These differences can impact infants' later language outcomes (Nicholas \& Geers, 2006; Szagun, 2001; 2004), and they could further inform the adaptations that mothers make to IDS in relation to their infants' individual perceptual and processing needs. We did not have access to this information in infants' medical records, so this is left as a 
direction for future research. As with many studies with infants with HL, we were unable to recruit infants from a narrow age range, or equal numbers of infants with unilateral and bilateral HL, or equal numbers of infants fitted with CI and Hearing Aids. Our analyses of these sub-groups suggest that hearing configuration may impact IDS qualities to infants with HL, which calls for the inclusion of these factors in the design of future studies.

The finding here that infants with HL were exposed to IDS with similar acoustic properties to that to their NH peers suggests that despite degraded input pre-intervention, these infants with HL still receive exposure to IDS with features that should benefit their language acquisition. Nevertheless, differences persist, and these differences may have implications for speech intelligibility and later language development in this population. Further research that involves controlling for infants' residual hearing, HL configuration and fitting device is required to understand fully the source of these adjustments in mothers' IDS, and the potential implications for infants' language development.

\section{Conclusion}

This study has shown that the hearing status of the infant has an impact on mothers' IDS, and this in turn, relates to language development. The results suggest that, as a result of infants' impaired ability to discriminate vowels due to HL, mothers respond by providing greater clarity by producing greater separation between formants in case of vowel/a/, and reducing the separation between formants in case of $/ \mathrm{i} /$ and $/ \mathrm{u} /$. Thus, mothers adjust their IDS in response to their infant's linguistic competence and needs. Accordingly, as the less clear vowel production in IDS to infants with HL does not have a detrimental effect on their receptive vocabulary, it appears that mothers may provide different didactic cues to infants with HL, but nevertheless, didactic cues that are equally effective as those provided to $\mathrm{NH}$ infants. Overall, these findings suggest that individual components of IDS may play different attentional and linguistic roles depending on infants' linguistic competence and individual linguistic and perceptual needs. 


\section{Acknowledgements}

This research was supported by HEARing Cooperative Research Centre grant 82631, "The Seeds of Language Development", to the $3^{\text {rd }}$ author. The second author's work is supported by the Basque Government through the BERC 2018-2021 program and by the Spanish Ministry of Science and Innovation through the Ramon y Cajal Research Fellowship, PID2019-105528GA-I00. We would like to thank all the parents and infants for participating in the study, "The Shepherd Centre" in Sydney and Wollongong, "Hear and Say" in Brisbane for their help in recruitment of participants with HL, and Benjawan Kasisopa, Maria Christou-Ergos, Hana Zjakic and Scott O’Loughlin for their assistance with data collection. 


\section{References}

Adriaans, F., \& Swingley, D. (2017). Prosodic exaggeration within infant-directed speech: Consequences for vowel learnability. The Journal of the Acoustical Society of America, 141(5), 3070-3078.

Andruski, J. E., \& Kuhl, P. K. (1996, October). The acoustic structure of vowels in mothers' speech to infants and adults. In Proceeding of Fourth International Conference on Spoken Language Processing. ICSLP'96 (Vol. 3, pp. 1545-1548). IEEE.

Audibert, N., \& Falk, S. (2018, June). Vowel space and f0 characteristics of infant-directed singing and speech. In Proceedings of the 19th international conference on speech prosody (pp. 153-157).

Bates, D., Maechler, M., \& Bolker, B. (2013). Package lme4: linear mixed-effects models using S4 classes. Google Scholar.

Benasich, A. A., \& Tallal, P. (2002). Infant discrimination of rapid auditory cues predicts later language impairment. Behavioural brain research, 136(1), 31-49.

Benders, T. (2013). Mommy is only happy! Dutch mothers' realisation of speech sounds in infantdirected speech expresses emotion, not didactic intent. Infant Behavior and Development, 36(4), $847-862$.

Bergeson, T. R., Miller, R. J., \& McCune, K. (2006). Mothers' speech to hearing-impaired infants and children with cochlear implants. Infancy, 10(3), 221-240.

Bernstein Ratner, N. (1984). Patterns of vowel modification in mother-child speech. Journal of Child Language, 11(3), 557-578. 
Bernstein Ratner, N., \& Pye, C. (1984). Higher pitch in BT is not universal: Acoustic evidence from Quiche Mayan. Journal of child language, 11(3), 515-522.

Bloom, L. (1973). One word at a time: the use of single-word utterances before syntax. The Hague: Mouton

Boersma, P. P. G., \& Weenink, D. J. M. (1996). Praat: Doing Phonetics by Computer: Version 3.4. Instituut voor Fonetische Wetenschappen.

Boons, T., Brokx, J. P., Frijns, J. H., Peeraer, L., Philips, B., Vermeulen, A., ... \& Van Wieringen, A. (2012). Effect of pediatric bilateral cochlear implantation on language development. Archives of pediatrics \& adolescent medicine, 166(1), 28-34.

Bradlow, A. R., Torretta, G. M., \& Pisoni, D. B. (1996). Intelligibility of normal speech I: Global and fine-grained acoustic-phonetic talker characteristics. Speech communication, 20(3-4), 255-272.

Burnham, D., Kitamura, C., \& Vollmer-Conna, U. (2002). What's new, pussycat? On talking to babies and animals. Science, 296(5572), 1435-1435.

Burnham, D. K., Joeffry, S., \& Rice, L. (2010). Computer-and human-directed speech before and after correction. In Proceedings of the 13th Australasian International Conference on Speech Science and Technology, 14-16 December 2010, Melbourne, Australia (pp. 13-17).

Burnham, E. B., Wieland, E. A., Kondaurova, M. V., McAuley, J. D., Bergeson, T. R., \& Dilley, L. C. (2015). Phonetic modification of vowel space in storybook speech to infants up to 2 years of age. Journal of Speech, Language, and Hearing Research, 58(2), 241-253.

Castellanos, A., Benedí, J. M., \& Casacuberta, F. (1996). An analysis of general acoustic-phonetic features for Spanish speech produced with the Lombard effect. Speech Communication, 20(1-2), 23-35. 
Chong, S. C. F., Werker, J. F., Russell, J. A., \& Carroll, J. M. (2003). Three facial expressions mothers direct to their infants. Infant and Child Development: An International Journal of Research and Practice, 12(3), 211-232.

Cooper, R. P., \& Aslin, R. N. (1990). Preference for infant-directed speech in the first month after birth. Child development, 61(5), 1584-1595.

Cristia, A., \& Seidl, A. (2014). The hyperarticulation hypothesis of infant-directed speech. Journal of Child Language, 41(4), 913-934.

Dodane, C., \& Al-Tamimi, J. (2007, August). An acoustic comparison of vowel systems in adultdirected-speech and child-directed speech: Evidence from French, English \& Japanese. In 16th International Congress of Phonetics Sciences (pp. 6-10).

Eaves Jr, B. S., Feldman, N. H., Griffiths, T. L., \& Shafto, P. (2016). Infant-directed speech is consistent with teaching. Psychological review, 123(6), 758-771.

Eisenberg, L. S., Martinez, A. S., \& Boothroyd, A. (2004, November). Perception of phonetic contrasts in infants: Development of the VRASPAC. In International congress series(Vol. 1273, pp. 364367). Elsevier.

Englund, K. T. (2018). Hypoarticulation in infant-directed speech. Applied Psycholinguistics, 39(1), $67-87$.

Englund, K. T., \& Behne, D. M. (2005). Infant directed speech in natural interaction-Norwegian vowel quantity and quality. Journal of psycholinguistic research, 34(3), 259-280.

Fenson, L., Dale, P., Reznick, J. S., Thal, D., Bates, E., Hartung, J., ... \& Reilly, J. (1993). MacArthur Communicative Inventories: User's guide and technical manual. San Diego. 
Ferguson, S. H., \& Kewley-Port, D. (2002). Vowel intelligibility in clear and conversational speech for normal-hearing and hearing-impaired listeners. The Journal of the Acoustical Society of America, 112(1), 259-271.

Ferguson, S. H., \& Kewley-Port, D. (2007). Talker differences in clear and conversational speech: Acoustic characteristics of vowels. Journal of Speech, Language, and Hearing Research, 50(5), 1241-1255.

Fernald, A., \& Simon, T. (1984). Expanded intonation contours in mothers' speech to newborns. Developmental Psychology, 20, 104-113.

Fernald, A., Taeschner, T., Dunn, J., Papousek, M., de Boysson-Bardies, B., \& Fukui, I. (1989). A cross-language study of prosodic modifications in mothers' and fathers' speech to preverbal infants. Journal of child language, 16(3), 477-501.

Fernald, A. (2000). Speech to infants as hyperspeech: Knowledge-driven processes in early word recognition. Phonetica, 57(2-4), 242-254.

Fernald, A., Perfors, A., \& Marchman, V. A. (2006). Picking up speed in understanding: Speech processing efficiency and vocabulary growth across the 2nd year. Developmental psychology, 42(1), 98-116.

Fitzpatrick, E. M., Gaboury, I., Durieux-Smith, A., Coyle, D., Whittingham, J., \& Nassrallah, F. (2019). Auditory and language outcomes in children with unilateral hearing loss. Hearing research, 372, 42-51.

Fitzpatrick, E. M., Whittingham, J., \& Durieux-Smith, A. (2014). Mild bilateral and unilateral hearing loss in childhood: A 20-year view of hearing characteristics, and audiologic practices before and after newborn hearing screening. Ear and Hearing, 35(1), 10-18. 
Fitzpatrick, E., Grandpierre, V., Durieux-Smith, A., Gaboury, I., Coyle, D., Na, E., \& Sallam, N. (2015). Children with mild bilateral and unilateral hearing loss: Parents' reflections on experiences and outcomes. Journal of deaf studies and deaf education, 21(1), 34-43.

Galle, M. E., Apfelbaum, K. S., \& McMurray, B. (2015). The role of single talker acoustic variation in early word learning. Language Learning and Development, 11(1), 66-79.

Gerstman, L. (1968). Classification of self-normalized vowels. IEEE transactions on audio and electroacoustics, 16(1), 78-80.

Graf Estes, K., \& Hurley, K. (2013). Infant-directed prosody helps infants map sounds to meanings. Infancy, 18(5), 797-824.

Grieser, D. L., \& Kuhl, P. K. (1988). Maternal speech to infants in a tonal language: Support for universal prosodic features in motherese. Developmental psychology, 24(1), 14-20.

Hartman, K. M., Bernstein Ratner, N., \& Newman, R. S. (2017). Infant-directed speech (IDS) vowel clarity and child language outcomes. Journal of child language, 44(5), 1140-1162.

Hayashi, A., Tamekawa, Y., \& Kiritani, S. (2001). Developmental change in auditory preferences for speech stimuli in Japanese infants. Journal of Speech, Language, and Hearing Research, 44(6), 1189-2000.

Hazan, V., \& Baker, R. (2011). Acoustic-phonetic characteristics of speech produced with communicative intent to counter adverse listening conditions. The Journal of the Acoustical Society of America, 130(4), 2139-2152.

Houston, D. M., Ying, E. A., Pisoni, D. B., \& Kirk, K. I. (2001). Development of pre-word-learning skills in infants with cochlear implants. The Volta Review, 103(4), 303-306. 
Houston, D. M., Pisoni, D. B., Kirk, K. I., Ying, E. A., \& Miyamoto, R. T. (2003). Speech perception skills of deaf infants following cochlear implantation: A first report. International Journal of Pediatric Otorhinolaryngology, 67(5), 479-495.

Jarosz, A. F., \& Wiley, J. (2014). What are the odds? A practical guide to computing and reporting Bayes factors. The Journal of Problem Solving, 7(1), 2.

Jeffreys, H. (1998). The theory of probability. OUP Oxford.

Johnston, J. C., Durieux-Smith, A., Angus, D., O'Connor, A., \& Fitzpatrick, E. (2009). Bilateral paediatric cochlear implants: A critical review. International Journal of Audiology, 48(9), 601617.

Joint Committee on Infant Hearing. (2007). Year 2007 position statement: principles and guidelines for early hearing detection and intervention programs. Pediatrics, 120(4), 898-921.

Kalashnikova, M., Carignan, C., \& Burnham, D. (2017). The origins of babytalk: smiling, teaching or social convergence?. Royal Society open science, 4(8), 170306.

Kalashnikova, M., \& Burnham, D. (2018). Infant-directed speech from seven to nineteen months has similar acoustic properties but different functions. Journal of child language, 1-19.

Kalashnikova, M., Goswami, U., \& Burnham, D. (2018). Mothers speak differently to infants at-risk for dyslexia. Developmental science, $21(1)$, e12487.

Kalashnikova, M., Goswami, U., \& Burnham, D. (2020). Infant-directed speech to infants at risk for dyslexia: A novel cross-dyad design. Infancy, 25(3), 286-303.

Kalashnikova, M., Schwarz, I. C., \& Burnham, D. (2016). OZI: Australian English communicative development inventory. First Language, 36(4), 407-427. 
Kitamura, C., Thanavishuth, C., Burnham, D., \& Luksaneeyanawin, S. (2001). Universality and specificity in infant-directed speech: Pitch modifications as a function of infant age and sex in a tonal and non-tonal language. Infant behavior and development, 24(4), 372-392.

Kitamura, C., \& Burnham, D. (2003). Pitch and communicative intent in mother's speech: Adjustments for age and sex in the first year. Infancy, 4(1), 85-110.

Knoll, M., \& Uther, M. (2004). Motherese and Chinese: Evidence of acoustic changes in speech directed at infants and foreigners. The Journal of the Acoustical Society of America, 116(4), 25222522.

Koester, L. S. (1995). Face-to-face interactions between hearing mothers and their deaf or hearing infants. Infant Behavior and Development, 18(2), 145-153.

Kondaurova, M. V., Bergeson, T. R., \& Dilley, L. C. (2012). Effects of deafness on acoustic characteristics of American English tense/lax vowels in maternal speech to infants. The Journal of the Acoustical Society of America, 132(2), 1039-1049.

Kondaurova, M. V., \& Bergeson, T. R. (2011). The effects of age and infant hearing status on maternal use of prosodic cues for clause boundaries in speech. Journal of Speech, Language, and Hearing Research, 54(3), 740-754.

Kondaurova, M. V., Bergeson, T. R., \& Xu, H. (2013). Age-Related Changes in Prosodic Features of Maternal Speech to Prelingually Deaf Infants with Cochlear Implants. Infancy, 18(5), 825-848.

Kuhl, P. K., Andruski, J. E., Chistovich, I. A., Chistovich, L. A., Kozhevnikova, E. V., Ryskina, V. L., ... \& Lacerda, F. (1997). Cross-language analysis of phonetic units in language addressed to infants. Science, 277(5326), 684-686. 
Kuznetsova, A., Brockhoff, P. B., \& Christensen, R. H. B. (2017). 1merTest package: tests in linear mixed effects models. Journal of Statistical Software, 82(13), 1-26.

Lam, C., \& Kitamura, C. (2010). Maternal interactions with a hearing and hearing-impaired twin: Similarities and differences in speech input, interaction quality, and word production. Journal of Speech, Language, and Hearing Research, 53(3), 543-555.

Lam, C., \& Kitamura, C. (2012). Mommy, speak clearly: Induced hearing loss shapes vowel hyperarticulation. Developmental Science, 15(2), 212-221.

Lenth, R., Singmann, H., Love, J., Buerkner, P., \& Herve, M. (2019). Package “emmeans”: Estimated marginal means, aka least-squares means. The Comprehensive R Archive Network, 1-67.

Leong, V., Kalashnikova, M., Burnham, D., \& Goswami, U. (2014). Infant-directed speech enhances temporal rhythmic structure in the envelope. In Fifteenth Annual Conference of the International Speech Communication Association.

Liu, H. M., Kuhl, P. K., \& Tsao, F. M. (2003). An association between mothers' speech clarity and infants’ speech discrimination skills. Developmental science, 6(3), F1-F10.

Ma, W., Golinkoff, R. M., Houston, D. M., \& Hirsh-Pasek, K. (2011). Word learning in infant-and adult-directed speech. Language Learning and Development, 7(3), 185-201.

Macherey, O., \& Carlyon, R. P. (2014). Cochlear implants. Current Biology, 24(18), R878-R884.

Martin, A., Schatz, T., Versteegh, M., Miyazawa, K., Mazuka, R., Dupoux, E., \& Cristia, A. (2015). Mothers speak less clearly to infants than to adults: A comprehensive test of the hyperarticulation hypothesis. Psychological science, 26(3), 341-347. 
Martinez, A., Eisenberg, L., Boothroyd, A., \& Visser-Dumont, L. (2008). Assessing speech pattern contrast perception in infants: Early results on VRASPAC. Otology \& neurotology: official publication of the American Otological Society, American Neurotology Society [and] European Academy of Otology and Neurotology, 29(2), 183-188.

Makowski, D., Lüdecke, D., Ben-Shachar, M. S., Wilson, M. D., Bürkner, P. C., \& Mahr, T. (2020). Package 'bayestestR', Retrieved August 20, 2020, from https://easystats.github.io/bayestestR/

Mather, E., \& Plunkett, K. (2011). Same items, different order: Effects of temporal variability on infant categorization. Cognition, 119(3), 438-447.

McMurray, B., Kovack-Lesh, K. A., Goodwin, D., \& McEchron, W. (2013). Infant directed speech and the development of speech perception: Enhancing development or an unintended consequence?. Cognition, 129(2), 362-378.

Miyamoto, R. T., Houston, D. M., \& Bergeson, T. (2005). Cochlear implantation in deaf infants. The Laryngoscope, 115(8), 1376-1380.

Miyazawa, K., Shinya, T., Martin, A., Kikuchi, H., \& Mazuka, R. (2017). Vowels in infant-directed speech: More breathy and more variable, but not clearer. Cognition, 166, 84-93.

Moeller, M. P., \& Tomblin, J. B. (2015). An introduction to the outcomes of children with hearing loss study. Ear and Hearing, 36(0 1), 4s-13s.

Molfese, D. L., \& Molfese, V. J. (1985). Electrophysiological indices of auditory discrimination in newborn infants: The bases for predicting later language development?. Infant Behavior and Development, 8(2), 197-211.

Molfese, D. L. (2000). Predicting dyslexia at 8 years of age using neonatal brain responses. Brain and language, $72(3), 238-245$. 
Munhall, K. G., MacDonald, E. N., Byrne, S. K., \& Johnsrude, I. (2009). Talkers alter vowel production in response to real-time formant perturbation even when instructed not to compensate. The Journal of the Acoustical Society of America, 125(1), 384-390.

Narayan, C. R., \& McDermott, L. C. (2016). Speech rate and pitch characteristics of infant-directed speech: Longitudinal and cross-linguistic observations. The Journal of the Acoustical Society of America, 139(3), 1272-1281.

Nicholas, J. G., \& Geers, A. E. (2007). Will they catch up? The role of age at cochlear implantation in the spoken language development of children with severe to profound hearing loss. Journal of speech, language, and hearing research, 50(4), 1048-1062.

Pardo, J. S. (2006). On phonetic convergence during conversational interaction. The Journal of the Acoustical Society of America, 119(4), 2382-2393.

Peter, V., Kalashnikova, M., Santos, A., \& Burnham, D. (2016). Mature neural responses to infantdirected speech but not adult-directed speech in pre-verbal infants. Scientific reports, 6, 34273.

R Core Team (2017). R: A language and environment for statistical computing. R Foundation for Statistical Computing, Vienna, Austria. URL https://www.r-project.org/

Sarant, J., Harris, D., Bennet, L., \& Bant, S. (2014). Bilateral versus unilateral cochlear implants in children: a study of spoken language outcomes. Ear and Hearing, 35(4), 396-409.

Rattanasone, N. X., Burnham, D., \& Reilly, R. G. (2013). Tone and vowel enhancement in Cantonese infant-directed speech at 3, 6, 9, and 12 months of age. Journal of Phonetics, 41(5), 332-343.

Smiljanić, R., \& Bradlow, A. R. (2005). Production and perception of clear speech in Croatian and English. The Journal of the Acoustical Society of America, 118(3), 1677-1688. 
Smiljanić, R., \& Bradlow, A. R. (2009). Speaking and hearing clearly: Talker and listener factors in speaking style changes. Language and linguistics compass, 3(1), 236-264.

Song, J. Y., Demuth, K., \& Morgan, J. (2010). Effects of the acoustic properties of infant-directed speech on infant word recognition. The Journal of the Acoustical Society of America, 128(1), 389400.

Stern, D. N., Spieker, S., Barnett, R. K., \& MacKain, K. (1983). The prosody of maternal speech: Infant age and context related changes. Journal of child language, 10(1), 1-15.

Szagun, G. (2001). Language acquisition in young German-speaking children with cochlear implants: Individual differences and implications for conceptions of a 'sensitive phase'. Audiology and Neurotology, 6(5), 288-297.

Szagun, G. (2004, November). Individual differences in language acquisition by young children with cochlear implants and implications for a concept of 'sensitive phase'. In International Congress Series (Vol. 1273, pp. 308-311). Elsevier.

Tang, P., Xu Rattanasone, N., Yuen, I., \& Demuth, K. (2017). Phonetic enhancement of Mandarin vowels and tones: Infant-directed speech and Lombard speech. The Journal of the Acoustical Society of America, 142(2), 493-503.

Thiessen, E. D., Hill, E. A., \& Saffran, J. R. (2005). Infant-directed speech facilitates word segmentation. Infancy, 7(1), 53-71.

Trainor, L. J., Austin, C. M., \& Desjardins, R. N. (2000). Is infant-directed speech prosody a result of the vocal expression of emotion?. Psychological science, 11(3), 188-195. 
Tsao, F. M., Liu, H. M., \& Kuhl, P. K. (2004). Speech perception in infancy predicts language development in the second year of life: A longitudinal study. Child development, 75(4), 10671084.

Uther, M., Knoll, M. A., \& Burnham, D. (2007). Do you speak E-NG-LI-SH? A comparison of foreigner-and infant-directed speech. Speech Communication, 49(1), 2-7.

Välimaa, T., Kunnari, S., Laukkanen-Nevala, P., Lonka, E., \& National Clinical Research Team. (2018). Early vocabulary development in children with bilateral cochlear implants. International journal of language \& communication disorders, 53(1), 3-15.

van Wieringen, A., Boudewyns, A., Sangen, A., Wouters, J., \& Desloovere, C. (2019). Unilateral congenital hearing loss in children: Challenges and potentials. Hearing research, 372, 29-41.

Vanormelingen, L., De Maeyer, S., \& Gillis, S. (2016). A comparison of maternal and child language in normally-hearing and hearing-impaired children with cochlear implants. Language, Interaction and Acquisition, 7(2), 145-179.

Wieland, E. A., Burnham, E. B., Kondaurova, M., Bergeson, T. R., \& Dilley, L. C. (2015). Vowel space characteristics of speech directed to children with and without hearing loss. Journal of Speech, Language, and Hearing Research, 58(2), 254-267.

Wong, P., \& Ng, K. W. S. (2018). Testing the Hyperarticulation and Prosodic Hypotheses of ChildDirected Speech: Insights From the Perceptual and Acoustic Characteristics of Child-Directed Cantonese Tones. Journal of Speech, Language, and Hearing Research, 61(8), 1907-1925.

Xu, N., Burnham, D., Kitamura, C., \& Vollmer-Conna, U. (2013). Vowel hyperarticulation in parrot, dog-and infant-directed speech. Anthrozoös, 26(3), 373-380.

Zeng, F. G. (2004). Trends in cochlear implants. Trends in amplification, 8(1), 1-34. 
Zeng, F. G., Tang, Q., \& Lu, T. (2014). Abnormal pitch perception produced by cochlear implant stimulation. PloS one, 9(2), e88662.

Zhang, Y., Koerner, T., Miller, S., Grice-Patil, Z., Svec, A., Akbari, D., ... \& Carney, E. (2011). Neural coding of formant-exaggerated speech in the infant brain. Developmental science, 14(3), 566-581. 


\section{Supplementary Materials}

\section{Participants' demographic data}

Table I. Chronological (CA) and Hearing age (HA) at testing (months), HL degree and configuration, HL device and aetiology of $H L$ for infants with $H L$

\begin{tabular}{|c|c|c|c|c|c|}
\hline ID & CA (HA) & HL degree & Configuratio & Device & Aetiology \\
\hline 1 & $9.2(7.70)$ & mild/moderate & bilateral & Hearing Aids & connexion 26 \\
\hline 4 & $10.59(9.09)$ & mild/moderate & bilateral & Hearing Aids & genetic \\
\hline 5 & $9.4(6.60)$ & severe/profound & bilateral & Cochlear Implants & congenital \\
\hline 6 & $14.6(6.60)$ & R: mild & unilateral & $\mathrm{BCHA}^{*}$ & microtia \\
\hline 7 & $29.29(8.29)$ & / & bilateral & Hearing Aids & sensory neural \\
\hline 8 & $\begin{array}{l}23.83 \\
(21.83)\end{array}$ & mild & bilateral & Hearing Aids & unknown \\
\hline 9 & $12.06(7.06)$ & moderate/severe & bilateral & Hearing Aids & birth \\
\hline 10 & $8.28(5.78)$ & moderate & bilateral & Hearing Aids & sensorineural \\
\hline 11 & $7.17(5.17)$ & moderate & bilateral & Hearing Aids & unknown \\
\hline 12 & $\begin{array}{l}24.82 \\
(32.32)\end{array}$ & severe & bilateral & Cochlear Implants & $\begin{array}{l}\text { Enlarged vestibular } \\
\text { aqueducts }\end{array}$ \\
\hline 13 & $8.25(5.75)$ & mild/moderate & bilateral & Hearing Aids & unknown \\
\hline 14 & $\begin{array}{l}21.99 \\
(16.59)\end{array}$ & profound & bilateral & Cochlear Implants & $\begin{array}{l}\text { unknown (genetic } \\
\text { history) }\end{array}$ \\
\hline 15 & $\begin{array}{l}35.96 \\
(32.86)\end{array}$ & $\begin{array}{l}\mathrm{R}: \\
\text { moderate/severe }\end{array}$ & unilateral & BCHA & microtia and atretia \\
\hline 16 & $8.02(6.02)$ & R: moderate & unilateral & $\begin{array}{l}\text { BAHA }^{* *} \\
\text { softband }\end{array}$ & microtia and atretia \\
\hline 18 & $17.06(7.56)$ & moderate & bilateral & Phonak Sky V50 P & unknown \\
\hline 23 & $\begin{array}{l}15.45 \\
(15.45)\end{array}$ & & unilateral & Hearing Aid & unknown \\
\hline 24 & $\begin{array}{l}24.13 \\
(24.13)\end{array}$ & $\begin{array}{l}\text { L: } \\
\text { mild/moderate }\end{array}$ & unilateral & Unaided & unknown \\
\hline 25 & $\begin{array}{l}17.98 \\
(7.53)\end{array}$ & $\begin{array}{l}\mathrm{L} \text { : severe; } \mathrm{R} \text { : } \\
\text { profound }\end{array}$ & bilateral & $\begin{array}{l}\text { Cochlear Nucleus } 7 \\
\text { processor }\end{array}$ & connexion 26 \\
\hline 26 & $\begin{array}{l}10.52 \\
(10.52)\end{array}$ & R: unknown & unilateral & N/A & sensorineural \\
\hline 29 & $8.09(6.59)$ & $\begin{array}{l}\mathrm{L}: \\
\text { moderate/severe } \\
\mathrm{R}: \text { severe }\end{array}$ & bilateral & Hearing Aids & unknown \\
\hline
\end{tabular}


*mild/moderate - HL on the border of mild and moderate (around 39-40 dBHL); moderate/severe - HL on the border of moderate and severe (around 69-70 dBHL); severe/profound - HL on the border of severe and profound (around 89-90 dBHL); L - left ear; $\mathrm{R}$ - right ear.

Table II. Infants' ages (months) at the Session 1 and Session 2 for infants with HL

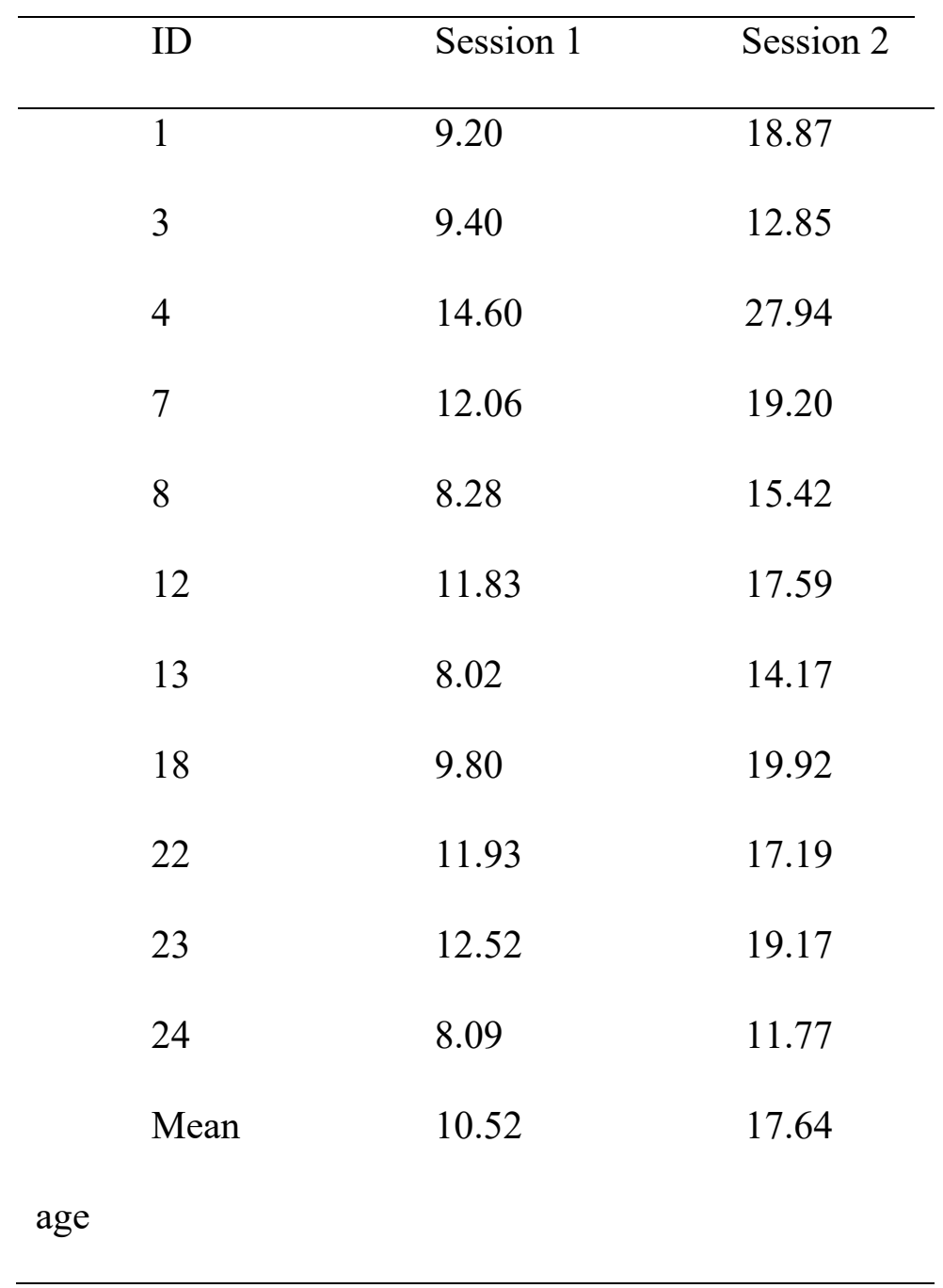




\section{Vowels and speech segments used in analyses in Experiment 1 and Experiment 2}

Table III. Detailed information about the vowels and speech segments extracted for vowel hyperarticulation and pitch height analyses in Experiments 1 and 2 (the values are Means and SDs are presented in parentheses).

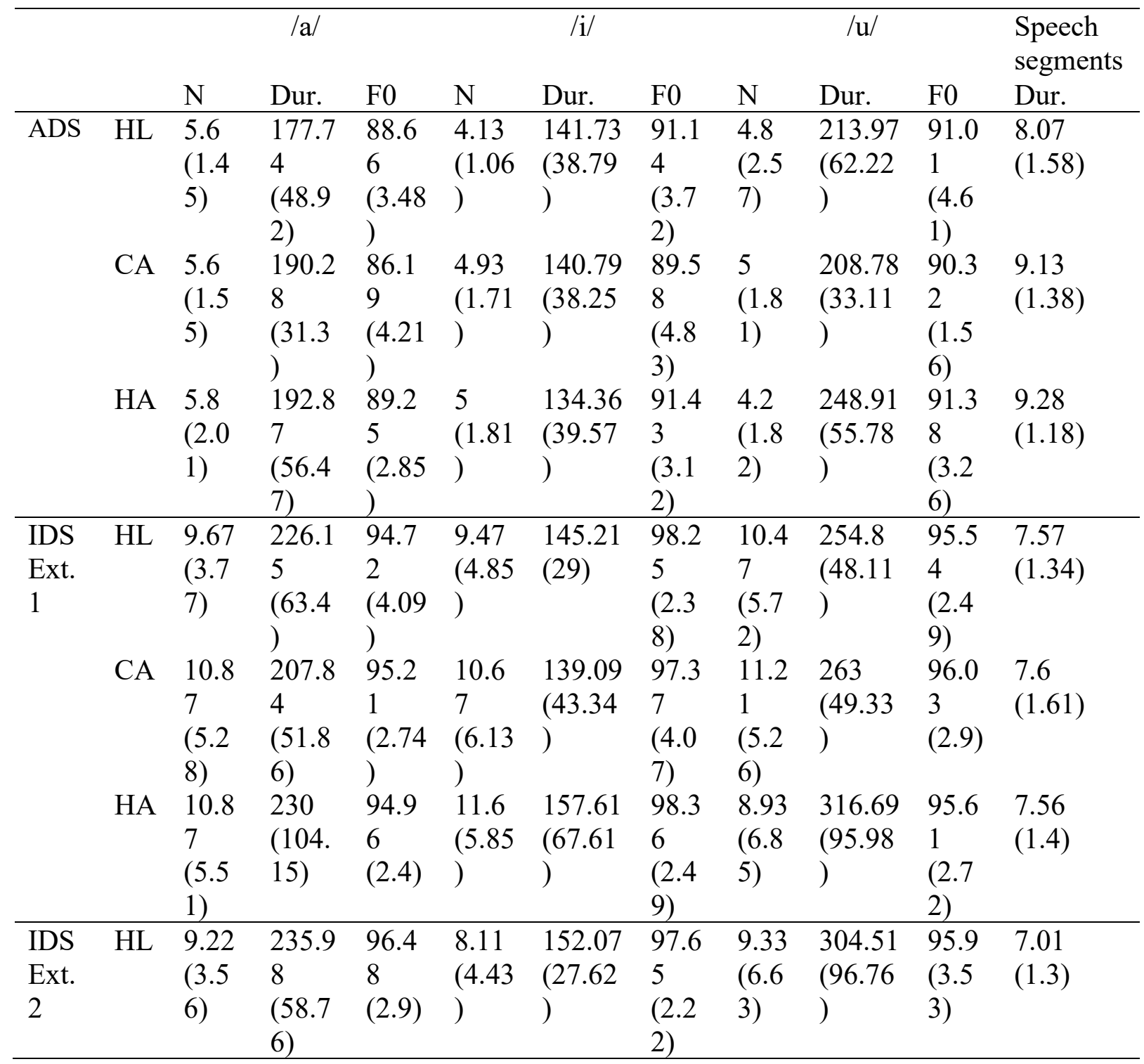




\section{Experiment 1}

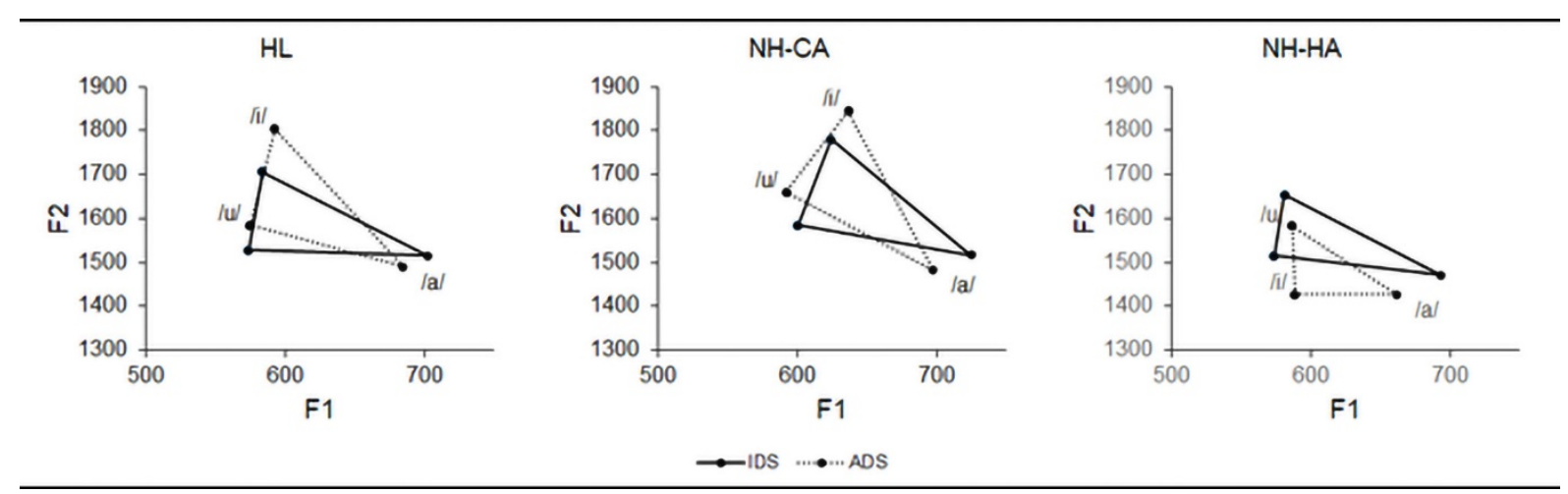

Figure 1. Vowel triangle areas for IDS and ADS for infants with HL, NH-CA, and NHHA.
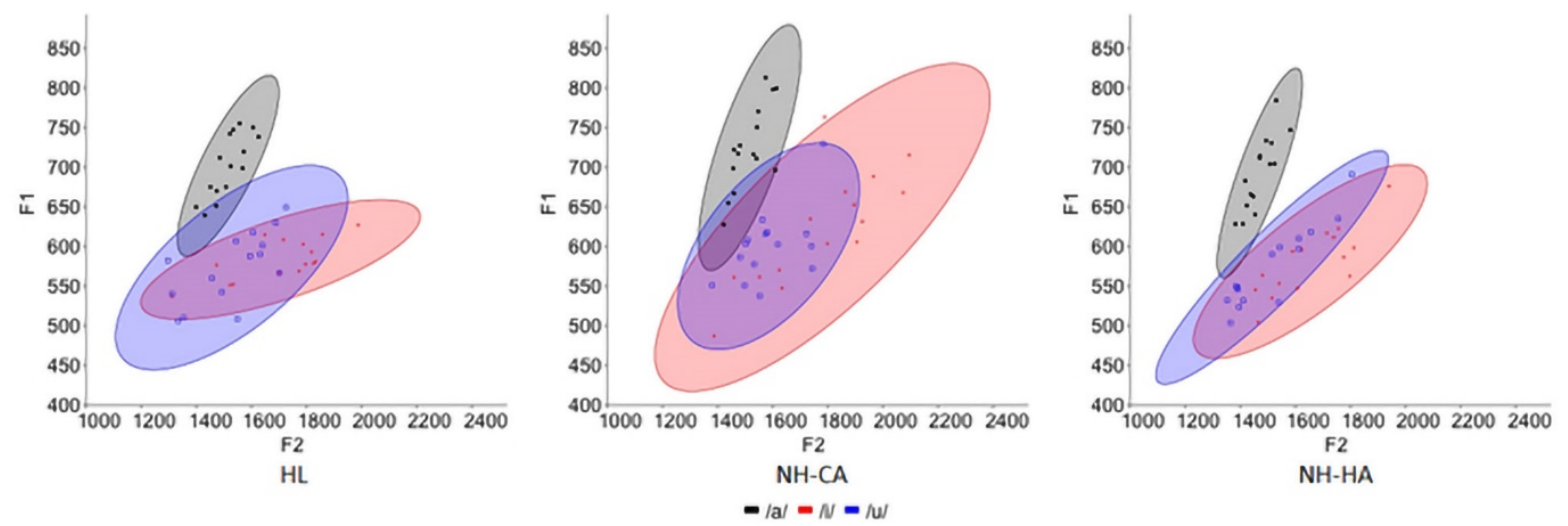

Figure 2. Distributions of vowels /a, i, u/ in IDS to HL, NH-CA and NH-HA infants (ellipses represent $95 \% \mathrm{CI}$ ). 


\section{Experiment 2}

Table IV. Summary of the LME model fitted for the variability in F2 for the vowels /a, $i, u /$ for infants with $H L$

\begin{tabular}{llllll}
\hline Fixed effects & Estimate & SE & df & t value & $p$ \\
\hline Intercept & 127.99 & 24.51 & 66.99 & 5.22 & $<.001$ \\
Session2 & 5.07 & 32.48 & 60.00 & .16 & $=.88$ \\
Vowel2 & 177.22 & 32.48 & 60.00 & 5.46 & $<.001$ \\
Vowel3 & 101.24 & 32.48 & 60.00 & 3.12 & $=.01$ \\
Session2:Vowel2 & 5.06 & 45.93 & 60.00 & .11 & $=.91$ \\
Session2:Vowel3 & -59.60 & 45.93 & 60.00 & -1.30 & $=.20$ \\
Random effects & & Variance & SD & \\
\hline Participants & 995.5 & 30.91 & \\
\hline
\end{tabular}

Table V. Summary of the LME model fitted for the F2-F1 distances for the vowels /a, $i$, u/ for infants with $H L$

\begin{tabular}{llllll}
\hline Fixed effects & Estimate & SE & df & t value & $\mathrm{p}$ \\
\hline Intercept & 794.82 & 25.60 & 32.43 & 31.05 & $<.001$ \\
Session2 & 9.94 & 30.62 & 586.32 & .32 & $=.75$ \\
Vowel2 & 349.10 & 30.62 & 583.03 & 11.40 & $<.001$ \\
Vowel3 & 174.33 & 28.80 & 585.01 & 6.05 & $<.001$ \\
Session2:Vowel2 & 36.18 & 46.95 & 581.34 & .77 & $=.44$ \\
Session2:Vowel3 & -13.91 & 45.51 & 583.03 & -.31 & $=.76$ \\
Random effects & & Variance & & SD & \\
\hline Participants & & 3016 & 54.92 & \\
\hline
\end{tabular}


11 months

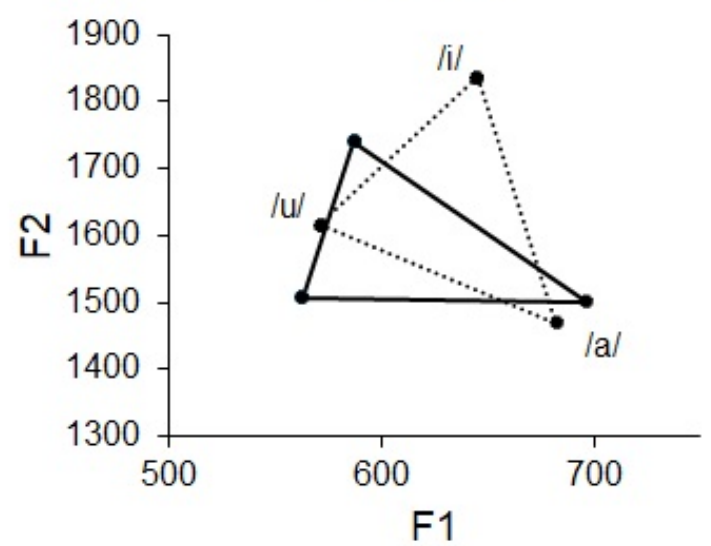

18 months

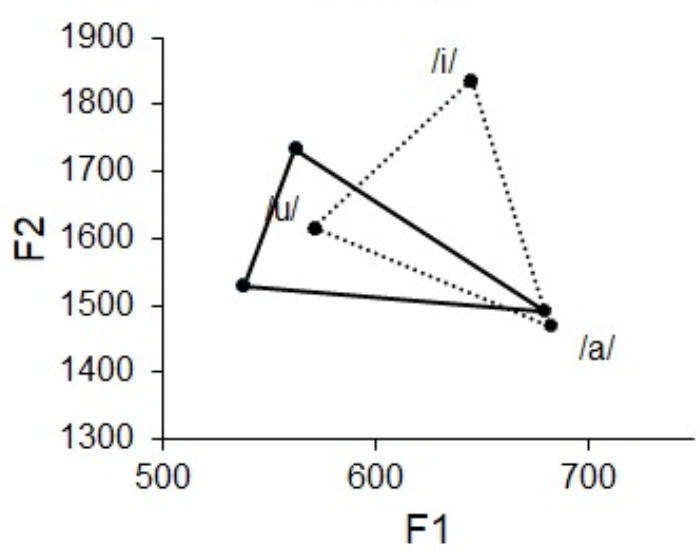

$\rightarrow$ IDS $\quad \cdots \cdot \cdots$ ADS

Figure 3. Vowel triangle areas for IDS and ADS for infants with HL at 11 and 18 months.

\section{Exploratory analyses}

\section{Individual differences in hearing configuration}

One-sample $t$-tests were used to compare vowel articulation and pitch hyper-scores to 1, and two separate univariate ANOVAs with hyper-scores as dependent variables and Group as a factor were conducted to assess the effects of hearing configuration on the hyper-scores (see Figure 4 for hyper-scores and Table VI for the summary of $t$-tests results).
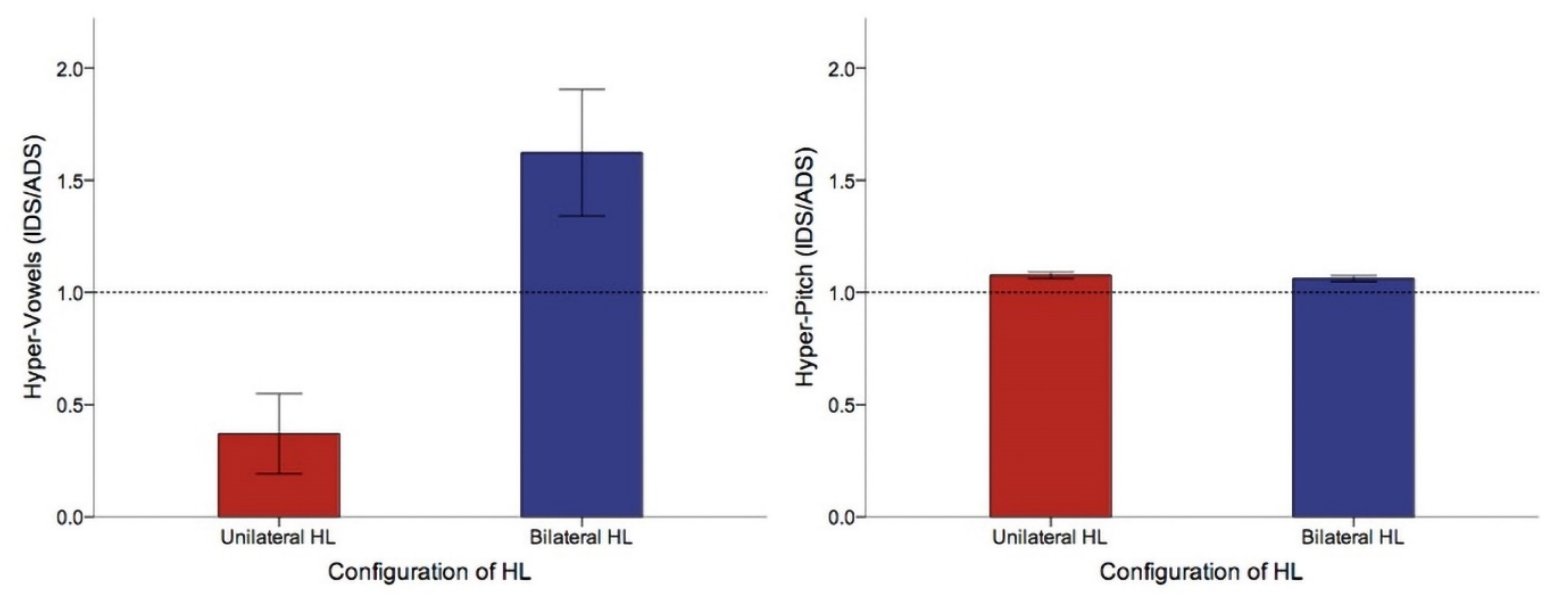
Figure 4. Hyper-scores for vowel articulation and mean pitch for infants with unilateral and bilateral HL (error bars represent SEM).

Vowel hyperarticulation. As can be seen in Table VI, mothers hyper-articulated vowels when addressing infants with bilateral HL, but hypo-articulated vowels in IDS to infants with unilateral HL. The univariate ANOVA yielded a significant effect of HL configuration, $F(1,13)=8.71, p=.01, \eta_{p}{ }^{2}=.40$ (see Figure 5 for vowel triangles). Thus, while mothers did not significantly hyperarticulate vowels to infants in the bilateral group (Table VI), the hyperarticulation index was significantly greater in the bilateral $(M=1.62, S D=.89)$ than in the unilateral group $(M=0.37, S D=.40), p=.01$.

Table VI. Results of one-sample t-test analyses (Cohen's d) comparing hyper-vowel and hyper-pitch scores to 1 ( $d f=4$ for unilateral group, $d f=9$ for bilateral group) in IDS to infants with unilateral and bilateral $H L$

\begin{tabular}{lll}
\hline Configuration & Hyper-vowels & Hyper-pitch \\
\hline Unilateral & $-3.53^{*}(1.58)$ & $5.42^{* *}(2.67)$ \\
Bilateral & $2.21(.70)$ & $4.78^{* *}(1.50)$ \\
\hline \multirow{2}{*}{$p=.02, * * p=.01$} &
\end{tabular}




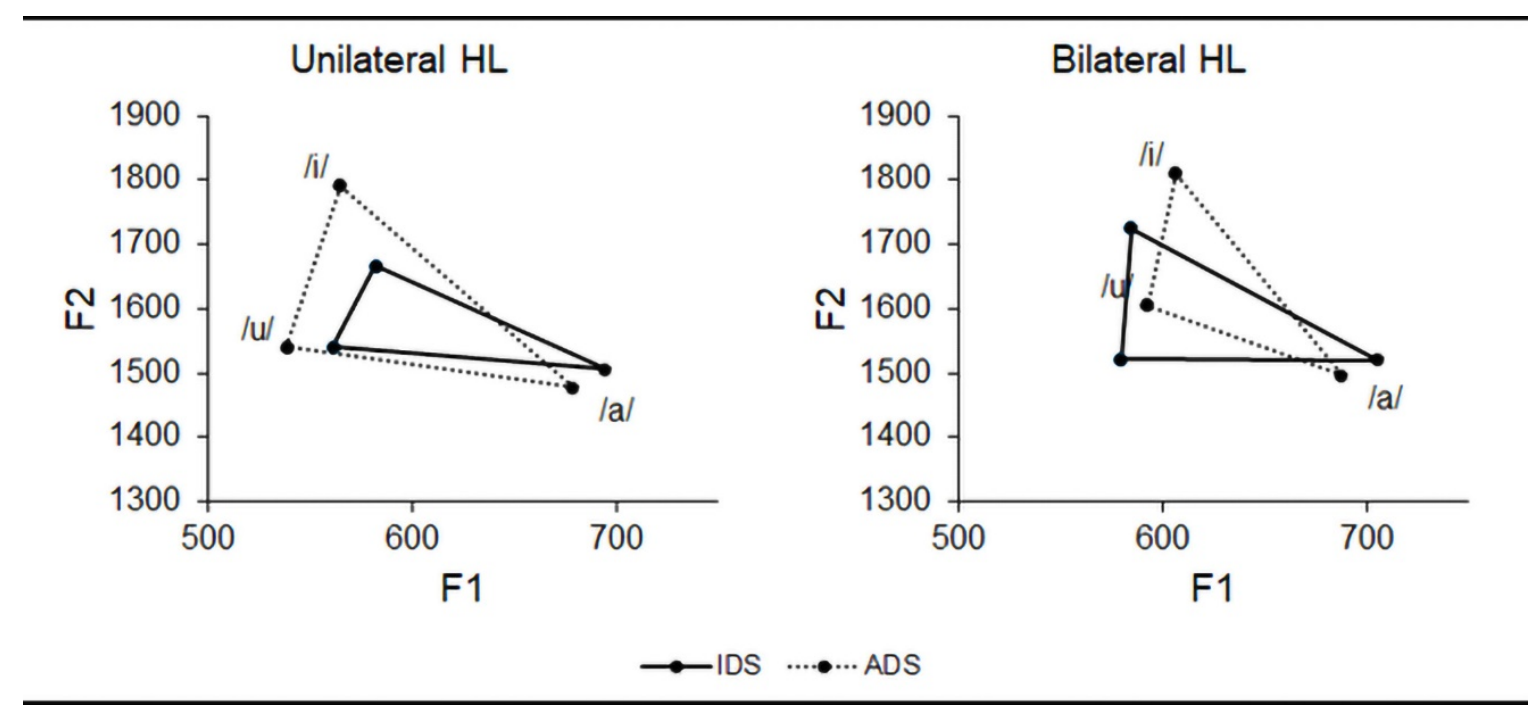

Figure 5. Vowel triangle areas for IDS and ADS for infants with unilateral and bilateral HL.

Pitch. Mothers produced exaggerated pitch in IDS to both groups of infants with HL, with no significant group effect $\left(F(1,13)=.47, p=.50, \eta_{p}{ }^{2}=.04\right)$.

\section{Individual differences in the degree of hearing loss}

Since it is possible that the degree of hearing loss can impact infants' language development, we conducted additional exploratory analyses comparing the acoustic components of IDS directed to infants with mild to moderate $(n=8)$ and severe to profound hearing loss $(n=7)$ in our sample.

Vowel hyperarticulation. The results showed that mothers did not expand or reduce their vowel space when addressing infants with mild/moderate and severe/profound hearing loss (Mild/moderate: $t(7)=.701, p=.506$, Cohen's $d=.248$; Severe/profound: $t(6)=.390, p=$ .710 , Cohen's $d=.147)$ and there was no significant difference between the two groups $(F(1$, $\left.13)=.085, p=.776, \eta_{p}^{2}=.006\right)$. 
Pitch. The $t$-tests indicated that mothers hyper-articulated pitch in IDS to both groups of infants (Mild/moderate: $t(7)=6.698, p=.01$, Cohen's $d=2.4$; Severe/profound: $t(6)=3.476$, $p=.01$, Cohen's $d=1.3)$, with no difference between the groups $(F(1,13)=1.358, p=.265$, $\left.\eta_{p}^{2}=.095\right)$

\section{Acoustic features in IDS to infants with Cochlear Implants and Hearing Aids}

Given that the type of intervention is one of the factors that can impact infants' language development (Bergeson, 2011; Wieland et al., 2015), we conducted additional exploratory analyses comparing the acoustic components of IDS directed to infants with Cochlear Implants $(n=4)$ and Hearing Aids $(n=9)$ in our sample.

Vowel hyperarticulation. The results showed that mothers did not expand or reduce their vowel space when addressing infants with Cochlear Implants $(t(3)=-65, p=.56$, Cohen's $d=.33)$ and Hearing Aids $(t(8)=1.86, p=1.0$, Cohen's $d=.62)$, and there was no significant difference between the two groups $\left(F(1,11)=2.458, p=.145, \eta_{p}^{2}=.183\right)$.

Pitch. The $t$-tests indicated that mothers hyper-articulated pitch in IDS to infants with Hearing Aids $(t(8)=5.70, p=.01$, Cohen's $d=1.50)$, but not in IDS to infants with Cochlear Implants $(t(3)=2.16, p=.01$, Cohen's $d=1.08)$. However, the group effect was not statistically significant $\left(F(1,11)=.223, p=.646, \eta_{p}^{2}=.020\right)$. 